\title{
Faster rumor spreading with multiple calls
}

\author{
Konstantinos Panagiotou \\ Ludwig-Maximilians-University \\ Munich, Germany \\ kpanagio@math.lmu.de
}

\author{
Ali Pourmiri \\ Max Planck Institute for Informatics \\ Saarbrücken, Germany \\ pourmiri@mpi-inf.mpg.de
}

\author{
Thomas Sauerwald \\ University of Cambridge \\ Cambridge, United Kingdom \\ thomas.sauerwald@cl.cam.ac.uk
}

Submitted: Apr 25, 2014; Accepted: Dec 16, 2014; Published: Feb 9, 2015

Mathematics Subject Classifications: 68W20; 68M14; 05C90

\begin{abstract}
We consider the random phone call model introduced by Demers et al.,which is a well-studied model for information dissemination in networks. One basic protocol in this model is the so-called Push protocol that proceeds in synchronous rounds. Starting with a single node which knows of a rumor, every informed node calls in each round a random neighbor and informs it of the rumor. The Push-Pull protocol works similarly, but additionally every uninformed node calls a random neighbor and may learn the rumor from it.

It is well-known that both protocols need $\Theta(\log n)$ rounds to spread a rumor on a complete network with $n$ nodes. Here we are interested in how much the spread can be speeded up by enabling nodes to make more than one call in each round. We propose a new model where the number of calls of a node is chosen independently according to a probability distribution $R$. We provide both lower and upper bounds on the rumor spreading time depending on statistical properties of $R$ such as the mean or the variance (if they exist). In particular, if $R$ follows a power law distribution with exponent $\beta \in(2,3)$, we show that the Push-Pull protocol spreads a rumor in $\Theta(\log \log n)$ rounds. Moreover, when $\beta=3$, the PushPull protocol spreads a rumor in $\Theta\left(\frac{\log n}{\log \log n}\right)$ rounds.
\end{abstract}




\section{Contents}

1 Introduction 3

2 Notations and Preliminaries 5

3 Some Useful Facts for Power Law Distributions 6

4 Push Protocol $\quad 7$

5 Push Protocol with Bounded Mean and Bounded Variance 9

5.1 The Preliminary Phase . . . . . . . . . . . . . . . . . . . 11

5.2 The Middle Phase . . . . . . . . . . . . . . . . . . . . . . . . . . . . . . . . . . 11

5.3 The Final Phase . . . . . . . . . . . . . . . . . . . . . . . . 15

6 Push Protocol with Bounded Mean 19

7 Lower Bound for Push-Pull $\quad 19$

8 Push-Pull Protocol with Bounded Mean and Bounded Variance 21

9 Push-Pull Protocol with Power Law Distribution $2<\boldsymbol{\beta}<3 \quad 21$

9.1 Proof of the Upper Bound . . . . . . . . . . . . . . . . . 21

9.2 Proof of the Lower Bound . . . . . . . . . . . . . . . . . 25

10 Push-Pull Protocol with Power Law Distribution $\beta=328$

10.1 Proof of Lower Bound . . . . . . . . . . . . . . . . . . 28

10.2 Proof of Upper Bound . . . . . . . . . . . . . . . . . . 29

11 Generating a New $C_{u}^{t}$ in Each Round (Theorem 1.6) 32 


\section{Introduction}

Randomized rumor spreading is an important primitive for spreading information in networks. The goal is to spread a piece of information, the so-called rumor, from an arbitrary node to all the other nodes. Randomized rumor spreading protocols are based on the simple idea that every node picks a random neighbor and these two nodes are able to exchange information in that round. This paradigm ensures that the protocol is local, scalable, and robust against network failures (cf. $[13,15])$. Therefore, these protocols have been successfully applied in other contexts such as replicated databases [8], failure detection [30], resource discovery [23], load balancing [3], data aggregation [25], and analysis of the spread of computer viruses [2].

The most basic variant of randomized rumor spreading is the Push protocol. At the beginning, there is a single node who knows of some rumor. Then in each of the following rounds every informed node calls a random neighbor chosen independently and uniformly at random and informs it of the rumor. The Pull protocol is symmetric, here every uninformed node calls a random neighbor chosen independently and uniformly at random, and if that neighbor happens to be informed the node becomes informed. The Push-Pull protocol is simply the combination of both protocols. Most studies in randomized rumor spreading concern the runtime, which is the number of rounds required until the rumor reaches all other nodes, and the communication overhead, which is the total number of information exchanges, produced by these protocols (see e.g. [24]).

In one of the first papers in this area, Frieze and Grimmett [19] proved that if the underlying graph is a complete graph with $n$ nodes, then the runtime of Push is $\log _{2} n+$ $\log n \pm o(\log n)$ with high probability ${ }^{1}$, where $\log n$ denotes the natural logarithm of $n$. This result was later strengthened by Pittel [29]. For the standard Push-Pull protocol, Karp et al. [24] proved a runtime bound of $\log _{3} n+\mathcal{O}(\log \log n)$. In order to overcome the large number of $\Theta(n \log n)$ calls, Karp et al. also presented an extension of the Push-Pull protocol together with a termination mechanism that spreads a rumor in $\mathcal{O}(\log n)$ rounds using only $\mathcal{O}(n \log \log n)$ messages. Doerr and Fouz [9] proposed a new protocol using only Push calls that achieves a runtime of $(1+o(1)) \log _{2} n$ using only $O(n \cdot f(n))$ calls (and messages), where $f(n)$ is an arbitrarily slowly growing function.

Besides the complete graph, randomized rumor spreading protocols have been shown to be efficient also on other topologies. In particular, their runtime is at most logarithmic in $n$ for topologies ranging from basic networks, such as random graphs $[15,14,16]$ and hypercubes [15], random regular graphs $[1,17]$, graphs with constant conductance [27, 6, 20], constant weak conductance [4] or constant vertex expansion [22, 21], to more complex structures including preferential attachment graphs modeling social networks [5]. In particular, recent studies establishing a sub-logarithmic runtime on certain social network models $[10,11,18]$ raise the question whether it is possible to achieve a sub-logarithmic runtime also on the complete graph. In addition to analyses on static graphs, there are also studies on mobile geometric graphs, e.g., [7, 28].

\footnotetext{
${ }^{1}$ with probability $1-o(1)$ as $n \rightarrow \infty$. For simplicity, we sometimes omit the "with high probability" in the introduction.
} 
Since all aforementioned protocols require $\Theta(\log n)$ rounds to spread the rumor on a complete graph, we equip nodes with the possibility of calling more than one node in each round. Specifically, we assume that the power of a node $u$, denoted by $C_{u}$, is a random variable, which has the same distribution as a random variable $R$ with support on the positive integers and which is independent of $u$. In order to keep the overall communication cost small, we focus on distributions $R$ satisfying $\sum_{u \in V} C_{u}=\mathcal{O}(n)$ with high probability - in particular, $R$ has bounded mean. Our aim is to understand the impact of the distribution of $R$ on the runtime of randomized rumor spreading. In particular, we seek for conditions on $R$ which are necessary (and/or sufficient) for a sublogarithmic runtime.

Our first result concerns the Push protocol for the case where $R$ has bounded mean and bounded variance, which is the most basic setting. Let $T_{\text {total }}$ be the first round in which all nodes are informed.

Theorem 1.1. Consider the Push protocol and assume that $R$ is a distribution with $\mathbf{E}[R]=\mathcal{O}(1)$ and $\operatorname{Var}[R]=\mathcal{O}(1)$. Then $\left|T_{\text {total }}-\left(\log _{1+\mathbf{E}[R]} n+\log _{\mathbf{e}^{\mathbf{E}}[R]} n\right)\right|=o(\log n)$.

Note that by putting $R \equiv 1$, we retain the classic result by Frieze and Grimmett. Our next result addresses the case where we drop the assumption on the variance, and it provides a lower bound of $\Omega(\log n)$ on the number of rounds. Although this result is less precise than Theorem 1.1, it demonstrates that it is necessary to consider the Push-Pull protocol in order to achieve a sub-logarithmic runtime.

Theorem 1.2. Assume that $R$ is any distribution with $\mathbf{E}[R]=\mathcal{O}(1)$. Then with probability $1-o(1)$, the Push protocol needs at least $\Omega(\log n)$ rounds to inform all nodes.

We point out that the lower bound in Theorem 1.2 is tight up to constant factors, as the results in $[19,29]$ for the standard Push protocol imply an upper bound of $\mathcal{O}(\log n)$ rounds. We now consider the Push-Pull protocol and extend the lower bound of $\Omega(\log n)$ from Theorem 1.1.

Theorem 1.3. Assume that $R$ is any distribution with $\mathbf{E}[R]=\mathcal{O}(1)$ and $\operatorname{Var}[R]=\mathcal{O}(1)$. Then for any constant $\epsilon>0$, with probability $1-\epsilon$ the Push-Pull protocol needs at least $\Omega(\log n)$ rounds to inform all nodes.

Theorem 1.3 establishes that an unbounded variance is necessary to break the $\Omega(\log n)$ lower bound. An important distribution with bounded mean but unbounded variance is the power law distribution with exponent $\beta \leqslant 3$, i.e., there are constants $0<c_{1} \leqslant c_{2}$ such that $c_{1} z^{1-\beta} \leqslant \operatorname{Pr}[R \geqslant z] \leqslant c_{2} z^{1-\beta}$ for any $z \geqslant 1$, and $\operatorname{Pr}[R \geqslant 1]=1$. We are especially interested in power law distributions, because they are scale invariant and have been observed in a variety of settings. Our main result below shows that this natural distribution achieves a sublogarithmic runtime.

Theorem 1.4. Assume that $R$ is a power law distribution with $2<\beta<3$. Then the Push-Pull protocol informs all nodes in $\Theta(\log \log n)$ rounds with probability $1-o(1)$. 
Notice that if $R$ is a power law distribution with $\beta>3$, then Theorem 1.3 applies because the variance of $R$ is bounded. Hence our results reveal a dichotomy in terms of the exponent $\beta$ : if $2<\beta<3$, then the Push-Pull protocol finishes in $\mathcal{O}(\log \log n)$ rounds, whereas for $\beta>3$ the Push-Pull protocol finishes in $\Theta(\log n)$ rounds ${ }^{2}$. While a very similar dichotomy was shown in [18] for random graphs with a power law degree distribution, our result here concerns the spread of the rumor from one to all nodes.

In the case $\beta=3$ we show that the runtime is close to the one in the $\beta>3$ case.

Theorem 1.5. Assume that $R$ is a power law distribution with $\beta=3$. Then the Push-Pull protocol informs all nodes in $\Theta\left(\frac{\log n}{\log \log n}\right)$ rounds with probability $1-o(1)$.

Finally, we also argue that it is necessary that the $C_{u}$ 's are chosen once and for all at the beginning, and they are not updated in each round. Indeed, suppose we generate in the th round a new variable $C_{u}^{t}$, which is the number of calls made by $u$ in that round. Then we prove the following lower bound.

Theorem 1.6. Assume that $R$ is any distribution with $\mathbf{E}[R]=\mathcal{O}(1)$. Then with probability $1-o(1)$, the Push-Pull protocol needs $\Omega(\log n)$ rounds to inform all nodes.

\section{Notations and Preliminaries}

We introduce some notation that will be used throughout the paper without further reference. In our setting, the Push, Pull and Push-Pull protocols proceed like the classic ones except that in each round, every (un)informed node $u$ calls $C_{u}$ node(s) chosen independently and uniformly at random and sends (requests) the rumor. For any of these protocols, we let $\mathcal{I}_{t}$ be the set of informed nodes at the end of round $t$ and $\mathcal{U}_{t}$ the set of uninformed nodes. We write $\mathcal{V}=\mathcal{I}_{t} \cup \mathcal{U}_{t}$ for the vertex set of the graph, and we assume $|\mathcal{V}|=n$. The size of $\mathcal{I}_{t}$ and $\mathcal{U}_{t}$ is denoted by $I_{t}$ and $U_{t}$. We indicate the set of newly informed nodes in round $t+1$ by $\mathcal{N}_{t}$ and its size is $N_{t}$. Let $S_{t}$ be the number of Push calls in round $t+1$, so $S_{t}=\sum_{u \in \mathcal{I}_{t}} C_{u} \geqslant I_{t}$. Let us define $\mathcal{N}_{t}^{\text {Pull }}$ and $\mathcal{N}_{t}^{\text {Push }}$ to be the set of newly informed nodes by Pull and Push calls in round $t+1$, respectively. The size of $\mathcal{N}_{t}^{\text {Pull }}$ and $\mathcal{N}_{t}^{\text {Push }}$ are denoted by $N_{t}^{\text {Pull }}$ and $N_{t}^{\text {Push }}$. The size of every set divided by $n$ will be denoted by the corresponding small letter, so $i_{t}, n_{t}$ and $s_{t}$ are used to denote $I_{t} / n$, $N_{t} / n$, and $S_{t} / n$, respectively. Further, let

$$
\mathcal{L}(z):=\left\{u \in \mathcal{V}: C_{u} \geqslant z\right\} \quad \text { and set } \quad L(z)=\mathcal{L}(z)
$$

Moreover, let $\Delta=\max _{u \in \mathcal{V}} C_{u}$.

We will use extensively the following two concentration inequalities. The first one is a Chernoff-type bound.

\footnotetext{
${ }^{2}$ We do not consider the case $\beta \leqslant 2$, since then there exists at least one node with degree $\Omega(n)$ and the rumor is spread in constant time. Additionally, $\mathbf{E}[R]$ is no longer bounded.
} 
Theorem 2.1 ([12]). Suppose that $X_{1}, X_{2} \ldots, X_{n} \in\{0,1\}$ are independent and identically distributed random variables and let $X:=\sum_{i=1}^{n} X_{i}$. Then for any $\lambda \geqslant 0$

$$
\operatorname{Pr}[|X-\mathbf{E}[X]| \geqslant \lambda] \leqslant 2 \cdot \mathrm{e}^{-\frac{\lambda^{2}}{2(\mathbf{E}[X]+\lambda / 3)}}
$$

In particular,

$$
\operatorname{Pr}[|X-\mathbf{E}[X]| \geqslant \mathbf{E}[X] / 2] \leqslant 2 \cdot \mathrm{e}^{-\frac{\mathbf{E}[X]^{2}}{8(\mathbf{E}[X]+\mathbf{E}[X] / 6)}}<2 \cdot \mathrm{e}^{-\frac{\mathbf{E}[X]}{10}} .
$$

The next inequality is known as the Bounded Difference inequality.

Theorem $2.2([26])$. Suppose that $X_{1}, X_{2} \ldots, X_{n}$ are independent random variables and every $X_{i}, 1 \leqslant i \leqslant n$, takes a value from a finite set $A_{i}$. Let $f: \prod_{1 \leqslant i \leqslant n} A_{i} \rightarrow \mathbb{R}$ be a real-valued function so that there exist $c_{1}, c_{2}, \ldots, c_{n}$ with

$\sup _{x_{1}, x_{2}, \ldots, x_{n}, x_{i}^{\prime}}\left|f\left(x_{1}, x_{2}, \ldots, x_{i}, \ldots, x_{n}\right)-f\left(x_{1}, x_{2}, \ldots, x_{i}^{\prime}, \ldots, x_{n}\right)\right| \leqslant c_{i}$, for every $1 \leqslant i \leqslant n$.

Then, for every $\lambda>0$,

$$
\operatorname{Pr}\left[\left|f\left(X_{1}, X_{2}, \ldots, X_{n}\right)-\mathbf{E}\left[f\left(X_{1}, X_{2}, \ldots, X_{n}\right)\right]\right| \geqslant \lambda\right] \leqslant 2 \cdot \mathrm{e}^{-\frac{\lambda^{2}}{2 \sum_{i=1}^{n} c_{i}^{2}}} .
$$

\section{Some Useful Facts for Power Law Distributions}

Let $R$ be a power law probability distribution with exponent $\beta$, i.e., there are constants $0<c_{1}<c_{2}$ so that for every integer $z \geqslant 1$,

$$
c_{1} \cdot z^{1-\beta} \leqslant \operatorname{Pr}\left[C_{u} \geqslant z\right] \leqslant c_{2} \cdot z^{1-\beta},
$$

and $\operatorname{Pr}\left[C_{u} \geqslant 1\right]=1$. In this section we collect some basic properties of $R$.

Fact 3.1. If $R$ is a power law distribution with $\beta>3$, then $\operatorname{Var}[R]=\mathcal{O}(1)$.

Proof. Since $\beta>3$

$$
\operatorname{Var}[R] \leqslant \mathbf{E}\left[R^{2}\right]=\sum_{z \geqslant 1} \operatorname{Pr}\left[R^{2} \geqslant z\right] \leqslant 1+\sum_{z \geqslant 2} \sqrt{c_{2} \cdot z^{1-\beta}}<\infty .
$$

Fact 3.2. Let $\beta>2$. Let $C_{u}, u \in \mathcal{V}$ be independent, power-law distributed random variables with exponent $\beta$. Then, with probability $1-o\left(\frac{1}{\log n}\right)$,

$$
\Delta:=\max _{u \in \mathcal{V}} C_{u} \leqslant n^{\frac{1}{\beta-1}} \cdot \log n .
$$


Proof. By definition,

$$
\operatorname{Pr}\left[C_{u} \geqslant n^{\frac{1}{\beta-1}} \log n\right] \leqslant c_{2} \cdot n^{-1} \cdot \log ^{1-\beta}(n) .
$$

Applying the union bound yields the claim.

Recall that $\mathcal{L}(z):=\left\{u \in \mathcal{V}, C_{u} \geqslant z\right\}$ and $L(z):=|\mathcal{L}(z)|$.

Proposition 3.3. Let $\beta>2$. Let $C_{u}, u \in \mathcal{V}$ be independent, power-law distributed random variables with exponent $\beta$. Then, for every $z=\mathcal{O}\left(n^{\frac{1}{\beta-1}} / \log n\right)$, with probability $1-o(1 / n)$

$$
\frac{n \cdot c_{1} \cdot z^{1-\beta}}{2} \leqslant L(z) \leqslant \frac{3 \cdot n \cdot c_{2} \cdot z^{1-\beta}}{2} .
$$

Proof. For $u \in \mathcal{V}$ let $I_{u}$ be the indicator random variable for the event $C_{u} \geqslant z$. Since the $C_{u}$ 's are independent and identically distributed, so are the $I_{u}$ 's. By linearity of expectation

$$
n \cdot c_{1} \cdot z^{1-\beta} \leqslant \mathbf{E}[L(z)] \leqslant n \cdot c_{2} \cdot z^{1-\beta} .
$$

Applying Theorem 2.1 to the random variable $X:=\sum_{u \in \mathcal{V}} I_{u}$ yields that

$$
\operatorname{Pr}[|L(z)-\mathbf{E}[L(z)]|>\mathbf{E}[L(z)] / 2]<2 \cdot \mathrm{e}^{-\frac{\mathbf{E}[L(z)]}{10}} \leqslant 2 \cdot \mathrm{e}^{-\frac{n \cdot c_{1} \cdot z^{1-\beta}}{10}}
$$

Since $z=\mathcal{O}\left(n^{\frac{1}{\beta-1}} / \log n\right)$, the claim follows.

\section{Push Protocol}

In this section we will show two general lemmas for the Push protocol that are valid for any $R$ with support on the positive integers. They will be used when analyzing the Push and the Push-Pull protocols.

Lemma 4.1. Consider the Push protocol and suppose that $S_{t} \leqslant \log ^{c} n$, where $c>0$ is an arbitrary constant. Then with probability at least $1-\mathcal{O}\left(n^{-1} \log ^{2 c} n\right)$ we have

$$
I_{t+1}=I_{t}+S_{t} .
$$

Proof. Recall that $S_{t}$ is the number of Push calls in round $t+1$. By applying the union bound, the probability that an informed node receives a call in round $t+1$ is bounded by $\frac{S_{t} I_{t}}{n}$. So, with probability at least $1-\frac{S_{t} I_{t}}{n}$, none of the calls are sent to a node in $\mathcal{I}_{t}$. Conditioning on this event, consider all calls one by one in an arbitrary order; then the probability that the $i$-th call informs a different node from the previous $i-1$ calls is $1-\frac{i-1}{U_{t}}$. Therefore the conditional probability that $S_{t}$ calls inform $S_{t}$ different nodes is at least

$$
\prod_{i=1}^{S_{t}-1}\left(1-\frac{i}{U_{t}}\right)>\left(1-\frac{S_{t}-1}{U_{t}}\right)^{S_{t}} \geqslant 1-\frac{S_{t}^{2}}{U_{t}}
$$


So the probability that $S_{t}$ calls inform $S_{t}$ different uninformed nodes is at least

$$
\left(1-\frac{S_{t} I_{t}}{n}\right) \cdot\left(1-\frac{S_{t}^{2}}{U_{t}}\right)=1-\mathcal{O}\left(\frac{S_{t}^{2}}{n}\right)
$$

where the above equality holds because $I_{t} \leqslant S_{t} \leqslant \log ^{c} n$ and $U_{t}=n(1-o(1))$. The claim follows.

Lemma 4.2. Consider the Push protocol. Then with probability at least $1-o\left(\frac{1}{\log n}\right)$

$$
s_{t}-2 s_{t}^{2}-2 \sqrt{\frac{s_{t} \log \log n}{n}} \leqslant n_{t} \leqslant s_{t} .
$$

Proof. Since $N_{t}$ is always bounded by $S_{t}, n_{t} \leqslant s_{t}$. To see the lower bound, let for $v \in \mathcal{U}_{t}$ $Z_{v}$ be the indicator random variable for the event $v \in \mathcal{I}_{t+1}$. Then $N_{t}=\sum_{v \in \mathcal{U}_{t}} Z_{v}$. Since the $Z_{v}$ 's are identically distributed random variables,

$$
\mathbf{E}\left[N_{t}\right]=U_{t} \cdot \operatorname{Pr}\left[Z_{v}=1\right] .
$$

Let $X_{i} \in \mathcal{V}, 1 \leqslant i \leqslant N=S_{t}$, denote the target of the $i$-th call. Define

$$
f\left(X_{1}, X_{2}, \ldots, X_{N}\right):=N_{t}
$$

to be the function counting the number of newly informed nodes in round $t+1$. Then $\mathbf{E}\left[f\left(X_{1}, X_{2}, \ldots, X_{N}\right)\right]=\mathbf{E}\left[N_{t}\right]$. For each change in just one coordinate of $f$, the following statement holds:

$$
\sup _{x_{1}, x_{2}, \ldots, x_{i}, x_{i}^{\prime} \in \mathcal{V}}\left|f\left(x_{1}, x_{2}, \ldots, x_{i}, \ldots, x_{N}\right)-f\left(x_{1}, x_{2}, \ldots, x_{i}^{\prime}, \ldots, x_{N}\right)\right| \leqslant 1 .
$$

Therefore by applying Theorem 2.2 , we obtain

$$
\operatorname{Pr}\left[\left|N_{t}-\mathbf{E}\left[N_{t}\right]\right| \geqslant \sqrt{4 \cdot S_{t} \cdot \log \log n}\right] \leqslant 2 \cdot \mathrm{e}^{-4 S_{t} \log \log n / 2 S_{t}}=o(1 / \log n) .
$$

So with probability $1-o(1 / \log n)$ we have

$$
N_{t}>\mathbf{E}\left[N_{t}\right]-2 \sqrt{S_{t} \log \log n}=U_{t} \cdot \operatorname{Pr}\left[Z_{v}=1\right]-2 \sqrt{S_{t} \log \log n} .
$$

Now we estimate $\operatorname{Pr}\left[Z_{v}=1\right]$. By the definition of Push

$$
\operatorname{Pr}\left[Z_{v}=1\right]=1-\prod_{u \in \mathcal{I}_{t}}\left(1-\frac{1}{n}\right)^{C_{u}} .
$$

Using that $1-x \leqslant \mathrm{e}^{-x} \leqslant 1-x+x^{2}$ for any $x \geqslant 0$

$$
\operatorname{Pr}\left[Z_{v}=1\right] \geqslant 1-\mathrm{e}^{-\sum_{u \in \mathcal{I}_{t}} C_{u} / n}=1-\mathrm{e}^{-s_{t}} \geqslant s_{t}-s_{t}^{2} .
$$


We now plug the value obtained by the above formula into (1) and normalize it. So we obtain

$$
\begin{aligned}
n_{t} & \geqslant\left(1-i_{t}\right) \cdot\left(s_{t}-s_{t}^{2}\right)-2 \sqrt{\frac{s_{t} \log \log n}{n}} \\
& =s_{t}-s_{t}^{2}-i_{t} \cdot\left(s_{t}-s_{t}^{2}\right)-2 \sqrt{\frac{s_{t} \log \log n}{n}} \geqslant s_{t}-2 s_{t}^{2}-2 \sqrt{\frac{s_{t} \log \log n}{n}},
\end{aligned}
$$

where the last inequality comes from the fact that $i_{t} \leqslant s_{t}$.

Corollary 4.3. Consider the Push protocol. Then with probability at least $1-o(1 / \log n)$ for any round $t$ in which $S_{t} \leqslant n / 8$ we have that $I_{t+1} \geqslant I_{t}+S_{t} / 2$.

Proof. If $1 \leqslant S_{t} \leqslant \log n$, then Lemma 4.1 yields that with probability $1-o(1 / \log n)$ we have $N_{t}=S_{t}$. If $\log n \leqslant S_{t} \leqslant n / 8$, then $2 s_{t}^{2} \leqslant s_{t} / 4$ and $2 \sqrt{s_{t} n^{-1} \log \log n} \leqslant s_{t} / 4$. Thus, Lemma 4.2 guarantees that with probability at least $1-o(1 / \log n)$

$$
n_{t} \geqslant s_{t}-2 s_{t}^{2}-2 \sqrt{\frac{s_{t} \log \log n}{n}} \geqslant \frac{s_{t}}{2} .
$$

Corollary 4.4. Consider the Push protocol. For any round $t$ and positive integer $k=$ $\mathcal{O}(\log n)$ in which $S_{t+k}=o(n)$, with probability $1-o\left(\frac{k}{\log n}\right)$

$$
I_{t+k} \geqslant I_{t} \cdot(3 / 2)^{k} .
$$

Proof. By assumption we have for every $1 \leqslant i \leqslant k$ that $S_{t+i}=o(n)$. Applying Corollary 4.3 shows that with probability $1-o\left(\frac{1}{\log n}\right)$

$$
I_{t+i} \geqslant I_{t+i-1}+\frac{S_{t+i-1}}{2} \geqslant I_{t+i-1} \cdot \frac{3}{2} \text {. }
$$

Using an inductive argument and the union bound for $k$ implies the statement.

\section{Push Protocol with Bounded Mean and Bounded Variance}

This section is devoted to the proof of Theorem 1.1. Recall that $T_{\text {total }}:=\min \left\{t \mid I_{t}=n\right\}$, i.e., the first round in which all nodes are informed. We claim that if $\mathbf{E}[R]=\mathcal{O}(1)$ and

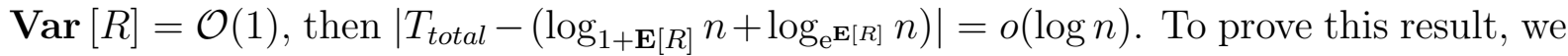
study the protocol in three consecutive phases. In the following we give a brief overview of the proof.

- The Preliminary Phase. This phase starts with just one informed node and ends when $I_{t} \geqslant \log ^{5} n$. Similar to the proof of the birthday paradox we show that in each round every Push call informs a different uninformed node and thus the number of informed nodes increases by $S_{t} \geqslant I_{t}$. Hence after $\mathcal{O}(\log \log n)$ rounds there are at least $\log ^{5} n$ informed nodes. Further, since $\mathbf{E}[R]=\mathcal{O}(1)$, after $\mathcal{O}(\log \log n)$ rounds we also have $S_{t} \leqslant \log ^{\mathcal{O}(1)} n$ for all these rounds. 
- The Middle Phase. This phase starts when $\log ^{5} n \leqslant I_{t} \leqslant S_{t} \leqslant \log ^{\mathcal{O}(1)} n$ and ends when $I_{t} \geqslant n / \log \log n$. First we show that the number of Push calls $S_{t}$ increases by a factor of approximately $1+\mathbf{E}[R]$ as long as the number of informed nodes is $o(n)$. Then we prove that the number of newly informed nodes in round $t+1$ is roughly the same as $S_{t}$. Therefore an inductive argument shows that it takes $\log _{1+\mathbf{E}[R]} n \pm o(\log n)$ rounds to reach $n / \log \log n$ informed nodes.

- The Final Phase. This phase starts when $I_{t} \geqslant \frac{n}{\log \log n}$ and ends when all nodes are informed with high probability. In this phase, we first prove that after $o(\log n)$ rounds the number of uninformed nodes decreases to $n / \log ^{5} n$. Then we show the probability that an arbitrary uninformed node remains uninformed is $e^{-\mathbf{E}[R] \pm o(1 / \log n)}$. Finally, an inductive argument establishes that it takes $\log _{\mathrm{e}^{\mathrm{E}}[R]} n \pm o(\log n)$ rounds until every node is informed.

In the following we present the detailed proofs for these phases. Before that we show the following proposition.

Proposition 5.1. Let $\epsilon>0$ and let $R$ be a random variable with support on the positive integers such that $\mathbf{E}[R]=\mathcal{O}(1)$ and $\operatorname{Var}[R]=\mathcal{O}(1)$. Let $\delta \geqslant 0$ be such that $U_{t}=n^{1-\delta}$, for some round $t$. Then, with probability $1-o(1 / \log n)$,

$$
\sum_{u \in \mathcal{U}_{t}} C_{u}=\mathcal{O}\left(n^{1-\delta / 2} \cdot \log ^{1+\epsilon} n\right)
$$

Proof. For $k \in \mathbb{N}$ let us define a random variable

$$
W_{k}:=\sum_{u \in \mathcal{V}} C_{u} \cdot \mathbb{1}\left(C_{u} \geqslant k\right)
$$

By linearity of expectation

$$
\begin{aligned}
\mathbf{E}\left[W_{k}\right] & =\sum_{u \in \mathcal{V}} \mathbf{E}\left[C_{u} \mathbb{1}\left(C_{u} \geqslant k\right)\right] \\
& =n \cdot \mathbf{E}\left[C_{u} \mathbb{1}\left(C_{u} \geqslant k\right)\right]=n \cdot \sum_{l \geqslant k} l \cdot \operatorname{Pr}\left[C_{u}=l\right] \leqslant \frac{n}{k} \cdot \sum_{l \geqslant k} l^{2} \cdot \operatorname{Pr}\left[C_{u}=l\right] .
\end{aligned}
$$

Since $C_{u}$ has bounded variance the last sum is in $\mathcal{O}(1)$. Thus, $\mathbf{E}\left[W_{k}\right]=\mathcal{O}(n / k)$. Markov's inequality implies that with probability $1-o(1 / \log n), W_{k}=\mathcal{O}\left(n \cdot \log ^{1+\epsilon} n \cdot k^{-1}\right)$. If we set $k=n^{\delta / 2}$, then

$$
\sum_{u \in \mathcal{U}_{t}} C_{u}=\sum_{\left\{u \in \mathcal{U}_{t}: C_{u} \geqslant k\right\}} C_{u}+\sum_{\left\{u \in \mathcal{U}_{t}: C_{u}<k\right\}} C_{u} \leqslant W_{k}+\mathcal{O}\left(n^{1-\delta} \cdot k\right)=\mathcal{O}\left(n^{1-\delta / 2} \cdot \log ^{1+\epsilon} n\right) .
$$




\subsection{The Preliminary Phase}

This phase starts with one informed node and ends when $I_{t} \geqslant \log ^{5} n$ and $S_{t} \leqslant \log ^{\mathcal{O}(1)} n$. Let $T_{0}$ be the first round in which the number of informed nodes exceeds $\log ^{5} n$.

Lemma 5.2. For any $t=\mathcal{O}(\log \log n)$, with probability at least $1-\log ^{-3} n$, we have $S_{t}=\log ^{\mathcal{O}(1)} n$.

Proof. We will bound the expected number of calls in each round $t$ as follows:

$$
\mathbf{E}\left[S_{t} \mid S_{t-1}\right]=S_{t-1}+\mathbf{E}\left[\sum_{u \in \mathcal{N}_{t-1}} C_{u} \mid S_{t-1}\right]=S_{t-1}+N_{t-1} \cdot \mathbf{E}[R] \leqslant S_{t-1} \cdot(1+\mathbf{E}[R]),
$$

where the last inequality comes from the fact that $N_{t-1} \leqslant S_{t-1}$. Since the origin of the rumor is chosen before determining the $C_{u}$ 's we have $\mathbf{E}\left[S_{0}\right]=\mathbf{E}[R]$. Applying the law of total expectation yields

$$
\mathbf{E}\left[S_{t}\right]=\mathbf{E}\left[\ldots \mathbf{E}\left[\mathbf{E}\left[S_{t} \mid S_{t-1}\right] \mid S_{t-2}\right] \ldots \mid S_{0}\right] \leqslant(1+\mathbf{E}[R])^{t} \mathbf{E}\left[S_{0}\right]=(1+\mathbf{E}[R])^{t} \mathbf{E}[R] .
$$

By using Markov's inequality we have that

$$
\operatorname{Pr}\left[S_{t} \geqslant(1+\mathbf{E}[R])^{t} \cdot \mathbf{E}[R] \cdot \log ^{3} n\right] \leqslant \log ^{-3} n .
$$

and the claim follows for any $t=\mathcal{O}(\log \log n)$.

Corollary 5.3. With probability $1-o(1)$ we have $T_{0}=\mathcal{O}(\log \log n)$.

Proof. Lemma 5.2 asserts that with probability at least $1-\mathcal{O}\left(\log ^{-3} n\right), S_{t}=\log ^{\mathcal{O}(1)} n$ for any $t=\mathcal{O}(\log \log n)$. Conditioning on this event, Lemma 4.1 guarantees that with probability $1-\left(n^{-1} \log ^{\mathcal{O}(1)} n\right)$, for any $t=\mathcal{O}(\log \log n)$,

$$
I_{t+1}=I_{t}+S_{t} \geqslant 2 I_{t}
$$

where the inequality comes from the fact that $S_{t} \geqslant I_{t}$. So, with probability at least

$$
\left(1-\frac{1}{\log ^{3} n}\right)\left(1-\mathcal{O}(\log \log n) \cdot \frac{\log ^{\mathcal{O}(1)} n}{n}\right)=1-o(1),
$$

there exists a round $T_{0}=\mathcal{O}(\log \log n)$ such that $I_{T_{0}} \geqslant \log ^{5} n$ and $S_{T_{0}} \leqslant \log ^{\mathcal{O}(1)} n$.

\subsection{The Middle Phase}

The phase starts when $\log ^{5} n \leqslant I_{t} \leqslant S_{t} \leqslant \log ^{\mathcal{O}(1)} n$ and ends when $I_{t} \geqslant n / \log \log n$. Let $T_{1}$ be the first round so that $I_{T_{1}} \geqslant n / \log \log n$. The main result of this subsection is that $\left|T_{1}-\log _{1+\mathbf{E}[R]} n\right|=o(\log n)$ with high probability. 
Lemma 5.4. Suppose for a round $t$ we have $s_{t}=\Omega\left(n^{-1} \cdot \log ^{5} n\right)$ and $s_{t}=o(1)$. Then for any $k=\mathcal{O}(\log n)$ with $(1+\mathbf{E}[R])^{k} s_{t}=o(1)$, with probability $1-o(k / \log n)$,

$$
\text { for all } 1 \leqslant i \leqslant k, \quad(1+\mathbf{E}[R])^{i} \cdot s_{t} \cdot(1-o(1)) \leqslant s_{t+i} \leqslant(1+\mathbf{E}[R])^{i} \cdot s_{t} \cdot(1+o(1)) .
$$

Proof. Consider the random variable $\sum_{u \in \mathcal{N}_{t}} C_{u}$. Since $\mathcal{N}_{t}$ is fixed and the random variables $C_{u}, u \in \mathcal{N}_{t}$ are independent we obtain that $\mathbf{E}\left[\sum_{u \in \mathcal{N}_{t}} C_{u}\right]=N_{t} \cdot \mathbf{E}[R]$. Moreover,

$$
\operatorname{Var}\left[\sum_{u \in \mathcal{N}_{t}} C_{u}\right]=N_{t} \cdot \operatorname{Var}[R]
$$

Chebychev's inequality implies that

$$
\operatorname{Pr}\left[\left|\sum_{u \in \mathcal{N}_{t}} C_{u}-N_{t} \mathbf{E}[R]\right| \geqslant \sqrt{N_{t} \log ^{2} n}\right] \leqslant \frac{N_{t} \operatorname{Var}[R]}{N_{t} \log ^{2} n}=o\left(\frac{1}{\log n}\right) .
$$

Since $S_{t+1}=S_{t}+\sum_{u \in \mathcal{N}_{t}} C_{u}$, it follows that with probability $1-o(1 / \log n)$,

$$
S_{t}+N_{t} \cdot \mathbf{E}[R]-\sqrt{N_{t} \log ^{2} n} \leqslant S_{t+1} \leqslant S_{t}+N_{t} \cdot \mathbf{E}[R]+\sqrt{N_{t} \log ^{2} n} .
$$

Using the above formula and the fact that $N_{t} \leqslant S_{t}$ we have

$$
S_{t+1} \leqslant S_{t}+S_{t} \cdot \mathbf{E}[R]+\sqrt{S_{t} \log ^{2} n} \leqslant S_{t} \cdot\left(1+\mathbf{E}[R]+\sqrt{\frac{\log ^{2} n}{S_{t}}}\right)
$$

Since $S_{t}$ is a non-decreasing function in $t$ and $\log ^{5} n \leqslant I_{t} \leqslant S_{t}$, with probability 1 $o(1 / \log n)$

$$
s_{t+1} \leqslant s_{t} \cdot(1+\mathbf{E}[R])\left(1+\sqrt{\frac{\log ^{2} n}{(1+\mathbf{E}[R])^{2} \log ^{5} n}}\right)<s_{t} \cdot(1+\mathbf{E}[R])\left(1+\frac{1}{\log ^{\frac{3}{2}} n}\right) .
$$

An inductive argument and the union bound for all $k$ events that violate the above inequality shows that for any $k=\mathcal{O}(\log n)$ with probability $1-o(k / \log n)$,

$$
\text { for all } 1 \leqslant i \leqslant k, \quad s_{t+i} \leqslant s_{t} \cdot(1+\mathbf{E}[R])^{i}(1+o(1)) \text {. }
$$

In order to prove the left hand side of (2), we use Lemma 4.2 which states with probability $1-o(1 / \log n)$,

$$
n_{t} \geqslant s_{t}-2 s_{t}^{2}-2 \sqrt{\frac{s_{t} \log \log n}{n}}
$$


Using the lower bound in (3) and the above formula implies that with probability 1 $o(1 / \log n)$,

$$
\begin{aligned}
s_{t+1} & \geqslant s_{t}+n_{t} \cdot \mathbf{E}[R]-\sqrt{\frac{n_{t} \log ^{2} n}{n}} \\
& \geqslant s_{t}+s_{t} \cdot \mathbf{E}[R]-2 s_{t}^{2} \cdot \mathbf{E}[R]-2 \sqrt{\frac{s_{t} \log \log n}{n}} \cdot \mathbf{E}[R]-\sqrt{\frac{s_{t} \cdot \log ^{2} n}{n}} \\
& \geqslant(1+\mathbf{E}[R]) s_{t}-2 \mathbf{E}[R] s_{t}^{2}-2 \sqrt{\frac{s_{t} \log ^{2} n}{n}} \\
& \geqslant(1+\mathbf{E}[R]) s_{t}-F\left(s_{t}\right),
\end{aligned}
$$

where $F\left(s_{t}\right)=2 \mathbf{E}[R] s_{t}^{2}+2 \sqrt{n^{-1} \cdot s_{t} \cdot \log ^{2} n}$. An inductive argument and the union bound for all $k$ events that violate the above inequality show that for any integer $k$ for which $(1+\mathbf{E}[R])^{k} \cdot s_{t}=o(1)$ with probability $1-o(k / \log n)$,

$$
\text { for all } 1 \leqslant i \leqslant k, \quad s_{t+i} \geqslant(1+\mathbf{E}[R])^{i} s_{t}-\sum_{j=0}^{i-1}(1+\mathbf{E}[R])^{j} F\left(s_{t+i-j}\right) .
$$

Inequality (4) yields that with probability $1-o(k / \log n)$,

$$
\text { for all } 1 \leqslant i \leqslant k=\mathcal{O}(\log n), \quad s_{t+i} \leqslant a \cdot s_{t} \cdot(1+\mathbf{E}[R])^{i},
$$

where $a:=1+o(1) . F\left(s_{t}\right)$ is a non-decreasing function in $s_{t}$ and hence for any $k=\mathcal{O}(\log n)$ and $1 \leqslant j \leqslant k$,

$$
\begin{aligned}
F\left(s_{t+i-j}\right) & \leqslant F\left(a \cdot(1+\mathbf{E}[R])^{i-j} s_{t}\right) \\
& \leqslant 2 \mathbf{E}[R](1+\mathbf{E}[R])^{2(i-j)}\left(a \cdot s_{t}\right)^{2}+2(1+\mathbf{E}[R])^{\frac{i-j}{2}} \sqrt{\frac{a \cdot s_{t} \log ^{2} n}{n}} .
\end{aligned}
$$

Hence by combining the above inequality and (5), we conclude that for any integer $k$, where $(1+\mathbf{E}[R])^{k} s_{t}=o(1)$ and $k=\mathcal{O}(\log n)$ with probability $1-o(k / \log n)$, for all $1 \leqslant i \leqslant k$

$$
\begin{aligned}
& s_{t+i} \\
& \geqslant(1+\mathbf{E}[R])^{i} s_{t}-2 \mathbf{E}[R] \sum_{j=0}^{i-1}(1+\mathbf{E}[R])^{2 i-j}\left(c \cdot s_{t}\right)^{2}-2 \sum_{j=0}^{i-1}(1+\mathbf{E}[R])^{\frac{i+j}{2}} \sqrt{\frac{c \cdot s_{t} \log ^{2} n}{n}} \\
& \geqslant(1+\mathbf{E}[R])^{i} s_{t}-d_{1} \cdot(1+\mathbf{E}[R])^{2 i} s_{t}^{2}-d_{2} \cdot(1+\mathbf{E}[R])^{i} \cdot \sqrt{\frac{s_{t} \log ^{2} n}{n}} \\
& =(1+\mathbf{E}[R])^{i} s_{t} \cdot\left(1-d_{1} \cdot(1+\mathbf{E}[R])^{i} s_{t}-d_{2} \cdot \sqrt{\frac{\log ^{2} n}{s_{t} n}}\right),
\end{aligned}
$$


where $d_{1}$ and $d_{2}$ are constants which do not depend on $i$. Since $(1+\mathbf{E}[R])^{k} s_{t}=o(1)$ and $s_{t}=\Omega\left(\frac{\log ^{5} n}{n}\right)$, for any $1 \leqslant i \leqslant k$,

$$
s_{t+i} \geqslant(1+\mathbf{E}[R])^{i} \cdot s_{t} \cdot(1-o(1)) .
$$

Lemma 5.5. Suppose that $\frac{\log ^{5} n}{n} \leqslant i_{t} \leqslant s_{t} \leqslant \frac{\log ^{\mathcal{O}(1)} n}{n}$. Then for any $k=\mathcal{O}(\log n)$ with $(1+\mathbf{E}[R])^{k} s_{t}=o(1)$, with probability $1-o(1)$,

$$
i_{t}+f_{2} \cdot(1+\mathbf{E}[R])^{k} \cdot s_{t} \cdot(1-o(1)) \leqslant i_{t+k} \leqslant i_{t}+f_{1} \cdot(1+\mathbf{E}[R])^{k} \cdot s_{t} \cdot(1+o(1)),
$$

where $f_{1}>0$ and $f_{2}>0$ are constants.

Proof. It is easy to see that

$$
i_{t+k}=i_{t}+\sum_{i=0}^{k-1} n_{t+i} \leqslant i_{t}+\sum_{i=0}^{k-1} s_{t+i} .
$$

Applying Lemma 5.4 implies that for any integer $k$ for which $(1+\mathbf{E}[R])^{k} \cdot s_{t}=o(1)$, with probability $1-o\left(\frac{k}{\log n}\right)$ the following upper bound holds:

$$
\begin{aligned}
i_{t+k} \leqslant i_{t}+\sum_{i=0}^{k-1} s_{t+i} & \leqslant i_{t}+s_{t} \cdot(1+o(1)) \cdot \sum_{i=0}^{k-1}(1+\mathbf{E}[R])^{i} \\
& =i_{t}+f_{1} \cdot(1+\mathbf{E}[R])^{k} \cdot s_{t} \cdot(1+o(1))
\end{aligned}
$$

where $f_{1}>0$ is a constant. On the other hand, Lemma 4.2 yields that with probability $1-o\left(\frac{1}{\log n}\right)$,

$$
n_{t} \geqslant s_{t}-2 s_{t}^{2}-2 \sqrt{\frac{s_{t} \log \log n}{n}} .
$$

Another application of Lemma 5.4 shows that with probability $1-o\left(\frac{k}{\log n}\right)$, for all integers $1 \leqslant i \leqslant k$ in which $(1+\mathbf{E}[R])^{k} s_{t}=o(1)$ and $s_{t} \geqslant \frac{\log ^{5} n}{n}$,

$$
(1+\mathbf{E}[R])^{i} \cdot s_{t} \cdot(1-o(1)) \leqslant s_{t+i} \leqslant(1+\mathbf{E}[R])^{i} \cdot s_{t} \cdot(1+o(1)) .
$$

Using these two inequalities, as long as $(1+\mathbf{E}[R])^{k} s_{t}=o(1)$, we have with probability 


$$
\begin{aligned}
& \begin{array}{l}
1-o\left(\frac{k}{\log n}\right), \\
i_{t+k}=i_{t}+\sum_{i=0}^{k-1} n_{t+i} \\
\geqslant i_{t}+\sum_{i=0}^{k-1} s_{t+i}-\sum_{i=0}^{k-1}\left\{2 s_{t+i}^{2}+2 \sqrt{\frac{s_{t+i} \log \log n}{n}}\right\} \\
\geqslant i_{t}+(1-o(1)) \sum_{i=0}^{k-1}(1+\mathbf{E}[R])^{i} s_{t} \\
\quad-(2+o(1)) \sum_{i=0}^{k-1}\left\{(1+\mathbf{E}[R])^{2 i} s_{t}^{2}+(1+\mathbf{E}[R])^{i / 2} \sqrt{\frac{s_{t} \log \log n}{n}}\right\} \\
\geqslant i_{t}+f_{2} \cdot(1+\mathbf{E}[R])^{k} \cdot s_{t}-d \cdot\left((1+\mathbf{E}[R])^{2 k} s_{t}^{2}+(1+\mathbf{E}[R])^{k / 2} \sqrt{\frac{s_{t} \log \log n}{n}}\right) \\
\geqslant i_{t}+f_{2} \cdot(1+\mathbf{E}[R])^{k} \cdot s_{t} \cdot\left(1-f \cdot(1+\mathbf{E}[R])^{k} \cdot s_{t}-d \cdot(1+\mathbf{E}[R])^{-k / 2} \sqrt{\frac{\log \log n}{s_{t} n}}\right)
\end{array}
\end{aligned}
$$

where $f_{2}>0$ and $d>0$ are constants. Since $\frac{\log ^{5} n}{n} \leqslant i_{t} \leqslant s_{t}$, we obtain that

$$
i_{t+k} \geqslant i_{t}+f_{2} \cdot(1+\mathbf{E}[R])^{k} \cdot s_{t} \cdot(1-o(1)) .
$$

By combining equations (7) and (6) we infer that with probability $1-o\left(\frac{k}{\log n}\right)$,

$$
i_{t}+f_{2} \cdot(1+\mathbf{E}[R])^{k} \cdot s_{t} \cdot(1-o(1)) \leqslant i_{t+k} \leqslant i_{t}+f_{1} \cdot(1+\mathbf{E}[R])^{k} \cdot s_{t} \cdot(1+o(1)) .
$$

Corollary 5.6. With probability $1-o(1)$ we have $\left|T_{1}-\log _{1+\mathbf{E}[R]} n\right|=o(\log n)$.

Proof. Applying Corollary 5.3 shows that with probability $1-o(1), T_{0}=\mathcal{O}(\log \log n)$, where $T_{0}$ is the first round in which $\frac{\log ^{5} n}{n} \leqslant i_{T_{0}} \leqslant s_{T_{0}} \leqslant \frac{\log ^{\mathcal{O}(1)} n}{n}$. Now we can apply Lemma 5.5 and set $k=\log _{1+\mathbf{E}[R]} n-o(\log n)$ such that with probability at least $1-o(1)$ we have $\frac{1}{\log \log n} \leqslant i_{T_{0}+k} \leqslant \frac{A}{\log \log n}$, where $A>1$ is a constant. Then we conclude that with probability $1-o(1),\left|T_{1}-\log _{1+\mathbf{E}[R]} n\right|=o(\log n)$.

\subsection{The Final Phase}

This phase starts with at least $\frac{n}{\log \log n}$ informed nodes and ends when all nodes get informed. Let $T_{1}$ be the first round in which $I_{T_{1}} \geqslant \frac{n}{\log \log n}$ and let $T_{2}$ be the first round in which all nodes are informed with probability $1-o(1)$. We will show that with probability $1-o(1),\left|\left(T_{2}-T_{1}\right)-\log _{e^{\mathbf{E}[R]}} n\right|=o(\log n)$. 
Lemma 5.7. With probability $1-o(1)$,

$$
\left|\left(T_{2}-T_{1}\right)-\log _{e} \mathbf{E}[R] n\right|=o(\log n) .
$$

Proof. We define the indicator random variable $Z_{v}$ for every $v \in \mathcal{U}_{t}$ and any round $t \geqslant T_{1}$ :

$$
Z_{v}= \begin{cases}1 & \text { if } v \text { does not get informed in round } \mathrm{t}+1 \\ 0 & \text { otherwise }\end{cases}
$$

Thus,

$$
\mathbf{E}\left[U_{t+1} \mid U_{t}\right]=\mathbf{E}\left[\sum_{v \in \mathcal{U}_{t}} Z_{v}\right]=U_{t} \cdot \operatorname{Pr}\left[Z_{v}=1\right],
$$

where for simplicity we omit the conditioning of $U_{t+1}$ on $U_{t}$ when dealing with the $Z_{v}$ 's. Using the fact that $1-\frac{1}{n}=e^{-\frac{1}{n}-\mathcal{O}\left(\frac{1}{n^{2}}\right)}$, we can approximate the value $\operatorname{Pr}\left[Z_{v}=1\right]$ as follows,

$$
\begin{aligned}
\operatorname{Pr}\left[Z_{v}=1\right] & =\prod_{u \in \mathcal{I}_{t}}\left(1-\frac{1}{n}\right)^{C_{u}}=\prod_{u \in \mathcal{I}_{t}} e^{-\frac{C_{u}}{n}-\mathcal{O}\left(\frac{C_{u}}{n^{2}}\right)} \\
& =e^{-\sum_{u \in \mathcal{I}_{t}}\left(\frac{C_{u}}{n}+\mathcal{O}\left(\frac{C_{u}}{n^{2}}\right)\right)}=e^{-s_{t}-\mathcal{O}\left(\frac{s_{t}}{n}\right)} .
\end{aligned}
$$

Since $\frac{s_{t}}{n}=\mathcal{O}\left(\frac{1}{n}\right)$ for any round and $\mathrm{e}^{-\mathcal{O}\left(\frac{1}{n}\right)}=1-\mathcal{O}\left(\frac{1}{n}\right)$,

$$
\mathbf{E}\left[U_{t+1} \mid U_{t}\right]=U_{t} e^{-s_{t}} \cdot e^{-\mathcal{O}\left(\frac{1}{n}\right)}=U_{t} e^{-s_{t}}-\mathcal{O}\left(\frac{U_{t}}{n}\right) .
$$

Since for every $u, v \in \mathcal{U}_{t}$,

$$
\operatorname{Pr}\left[Z_{u}=1 \cap Z_{v}=1\right]=\operatorname{Pr}\left[Z_{u}=1 \mid Z_{v}=1\right] \cdot \operatorname{Pr}\left[Z_{v}=1\right] \leqslant \operatorname{Pr}\left[Z_{v}=1\right] \cdot \operatorname{Pr}\left[Z_{u}=1\right],
$$

we have that

$$
\mathbf{E}\left[Z_{u} \cdot Z_{v}\right] \leqslant \mathbf{E}\left[Z_{u}\right] \cdot \mathbf{E}\left[Z_{v}\right]
$$

Therefore,

$$
\begin{aligned}
\operatorname{Var}\left[\sum_{v \in \mathcal{U}_{t}} Z_{v}\right] & =\sum_{v \in \mathcal{U}_{t}} \mathbf{E}\left[Z_{v}^{2}\right]+\sum_{u \neq v}\left(\mathbf{E}\left[Z_{u} \cdot Z_{v}\right]-\mathbf{E}\left[Z_{u}\right] \cdot \mathbf{E}\left[Z_{v}\right]\right) \\
& \leqslant \sum_{v \in \mathcal{U}_{t}} \mathbf{E}\left[Z_{v}^{2}\right]=U_{t} \cdot \operatorname{Pr}\left[Z_{v}=1\right]=\mathbf{E}\left[U_{t+1} \mid U_{t}\right] \leqslant U_{t}
\end{aligned}
$$

Applying Chebychev's inequality implies that with probability $1-o\left(\frac{1}{\log n}\right)$,

$$
\left|U_{t+1}-\mathbf{E}\left[U_{t+1} \mid U_{t}\right]\right| \leqslant \sqrt{U_{t} \log ^{2} n} .
$$


Combining inequalities (8) and (9) yields that with probability $1-o\left(\frac{1}{\log n}\right)$,

$$
\left|U_{t+1}-U_{t} e^{-s_{t}}\right| \leqslant \sqrt{U_{t} \log ^{2} n}+\mathcal{O}\left(\frac{U_{t}}{n}\right) \leqslant 2 \sqrt{U_{t} \log ^{2} n} .
$$

According to the value of $U_{t}$, we consider two cases.

- Suppose that $U_{t} \geqslant \frac{n}{\log ^{5} n}$. Note that $s_{t} \geqslant i_{t} \geqslant \frac{1}{\log \log n}$ by the assumption of the lemma. Since $s_{t}$ is a non-decreasing value in $t$ and $U_{t}<n$ the recursive formula (10) implies that with probability $1-o\left(\frac{1}{\log n}\right)$,

$$
U_{t+1} \leqslant U_{t} \cdot e^{\frac{-1}{\log \log n}}+2 \sqrt{n \log ^{2} n} .
$$

Using an inductive argument shows that with probability $1-o\left(\frac{k}{\log n}\right)$,

$$
U_{t+k} \leqslant U_{t} \cdot e^{\frac{-k}{\log \log n}}+\sum_{i=0}^{k-1} e^{\frac{-i}{\log \log n}} \cdot\left(2 \sqrt{n \log ^{2} n}\right) .
$$

Hence after at most $k_{0}=6 \log \log ^{2} n$ rounds with probability $1-o(1)$ the number of uninformed nodes decreases to $\frac{n}{\log ^{6} n}+\mathcal{O}\left(\sqrt{n \log ^{2} n}\right)$, where $c>0$ is a constant.

- Suppose that $U_{t} \leqslant \frac{n}{\log ^{5} n}$. If we set $n^{\delta}=\log ^{5} n$, then applying Proposition 5.1 implies that for any $t$ for which $U_{t}=\mathcal{O}\left(\frac{n}{\log ^{5} n}\right)$ with probability $1-o\left(\frac{1}{\log n}\right)$,

$$
\sum_{u \in \mathcal{U}_{t}} C_{u}=o\left(\frac{n}{\log n}\right) \text {. }
$$

On the other hand, using Chebychev's inequality yields that with probability 1 $o\left(\frac{1}{\log n}\right)$,

$$
\left|\sum_{u \in \mathcal{V}} C_{u}-n \cdot \mathbf{E}[R]\right| \leqslant \sqrt{n \cdot \log ^{2} n} .
$$

Combining the above equality and equality (11) results into an approximation for $s_{t}$ which is not best possible but it suffices for our purpose. We know that

$$
s_{t}=\sum_{u \in \mathcal{V}} C_{u}-\sum_{u \in \mathcal{U}_{t}} C_{u}
$$

So,

$$
\mathbf{E}[R]-\sqrt{\frac{\log ^{2} n}{n}}-o\left(\frac{1}{\log n}\right) \leqslant s_{t} \leqslant \mathbf{E}[R]+\sqrt{\frac{\log ^{2} n}{n}} .
$$


Therefore, $s_{t}$ can be replaced by $\mathbf{E}[R] \pm o\left(\frac{1}{\log n}\right)$ with probability $1-o\left(\frac{1}{\log n}\right)$. Inequality (10) implies that

$$
\alpha \cdot U_{t}-2 \sqrt{U_{t} \log ^{2} n} \leqslant U_{t+1} \leqslant \alpha \cdot U_{t}+2 \sqrt{U_{t} \log ^{2} n}
$$

where $\alpha=e^{-\mathbf{E}[R] \pm o(1 / \log n)}$. So as long as $U_{t} \geqslant \log ^{5} n$ with probability $1-o\left(\frac{1}{\log n}\right)$,

$$
\begin{aligned}
U_{t+1} \leqslant \alpha \cdot U_{t}+2 \sqrt{U_{t} \log ^{2} n} & =\alpha \cdot U_{t}\left(1+2 \sqrt{\frac{\log ^{2} n}{\alpha^{2} U_{t}}}\right) \\
& \leqslant \alpha \cdot U_{t}\left(1+2 \sqrt{\frac{\log ^{2} n}{\alpha^{2} \log ^{5} n}}\right) \leqslant \alpha \cdot U_{t}\left(1+\frac{2}{\alpha \log ^{\frac{3}{2}} n}\right) .
\end{aligned}
$$

Now for any $k$ for which $U_{t} e^{-k \mathbf{E}[R]} \geqslant \log ^{5} n$, with probability $1-o\left(\frac{k}{\log n}\right)$,

$$
U_{t+k} \leqslant \alpha^{k} \cdot U_{t} \cdot\left(1+\frac{2}{\alpha \log ^{\frac{3}{2}} n}\right)^{k}=\alpha^{k} \cdot U_{t} \cdot(1+o(1)) .
$$

In order to lower bound $U_{t+k}$ we apply the lower bound (12) inductively. So we have that with probability $1-o\left(\frac{k}{\log n}\right)$,

$$
U_{t+k} \geqslant \alpha^{k} \cdot U_{t}-\sum_{i=0}^{k-1} 2 \cdot \alpha^{i} \cdot \sqrt{U_{t+k-i-1} \log ^{2} n} .
$$

Applying inequality (13) yields that with probability $1-o\left(\frac{k}{\log n}\right)$,

$$
\sqrt{U_{t+k-i} \log ^{2} n} \leqslant \alpha^{\frac{k-i}{2}} \cdot \sqrt{U_{t}(1+o(1)) \log ^{2} n} .
$$

Thus,

$$
\begin{aligned}
U_{t+k} & \geqslant \alpha^{k} \cdot U_{t}-(1+o(1)) \sum_{i=0}^{k-1} \alpha^{\frac{k-i-1}{2}} \cdot \sqrt{U_{t} \log ^{2} n} \\
& \geqslant \alpha^{k} \cdot U_{t}-c \cdot \alpha^{\frac{k}{2}} \cdot \sqrt{U_{t} \log ^{2} n}
\end{aligned}
$$

where $c>0$ is a constant and the last inequality holds because $\sum_{i=0}^{k-1} \alpha^{\frac{k-i-1}{2}}=$ $\mathcal{O}\left(\alpha^{\frac{k}{2}}\right)$. Combining the inequalities (13) and (14) yields for any $k$ satisfying

$$
U_{t} e^{-k \mathbf{E}[R]} \geqslant \log ^{5} n
$$

with probability $1-o\left(\frac{k}{\log n}\right)$, 


$$
\alpha^{k} \cdot U_{t}(1-o(1)) \leqslant U_{t+k} \leqslant \alpha^{k} \cdot U_{t}(1+o(1)) .
$$

Hence by taking $k=\log _{e^{\mathbf{E}[R]}} n-o(\log n)$, with probability $1-o(1)$, the number of uninformed nodes after $T_{1}+k_{0}+k$ rounds decreases to $\log ^{5} n$, so we have at most $\log ^{5} n$ uninformed nodes. Using the fact that for every $x \geqslant 0,1-x \leqslant e^{-x}$, the probability that a node does not get informed after $k_{1}$ additional rounds is bounded from above by

$$
\prod_{u \in \mathcal{I}_{t}}\left(1-\frac{1}{n}\right)^{C_{u} \cdot k_{1}} \leqslant e^{-k_{1} \sum_{u \in \mathcal{I}_{t}} C_{u}} .
$$

We already know that $s_{t}=\mathbf{E}[R] \pm o\left(\frac{1}{\log n}\right)$ and $s_{t}$ is an non-decreasing value in $t$ so

$$
\sum_{u \in \mathcal{I}_{t}} C_{u}=s_{t}>\frac{\mathbf{E}[R]}{2} .
$$

Thus the union bound implies that the probability that every node in $\mathcal{U}_{t}$ does not get informed is bounded by $\log ^{5} n \cdot e^{\frac{-k_{1} \cdot \mathbf{E}[R]}{2}}$. By choosing $k_{1}=\Theta(\log \log n)$ we conclude that with probability $1-o(1)$ all nodes get informed. So we have with probability at least $1-o(1)$ that $T_{2} \leqslant T_{1}+k_{0}+k+k_{1}$, and $k_{0}+k+k_{1}=\log _{e^{\mathrm{E}[R]}} n+o(\log n)$.

\section{Push Protocol with Bounded Mean}

This section is devoted to the proof of Theorem 1.2.

Proof. In the Push protocol, in round $t+1$, at most $S_{t}$ randomly chosen uninformed nodes are informed. This implies that $\mathbf{E}\left[S_{t+1} \mid S_{t}\right]$ increases by at most $\mathbf{E}[R] \cdot S_{t}$. Since the origin of the rumor is chosen without knowing $C_{u}, \mathbf{E}\left[S_{0}\right]=\mathbf{E}[R]$. Using the law of total expectation yields that

$$
\mathbf{E}\left[S_{t}\right]=\mathbf{E}\left[\ldots \mathbf{E}\left[\mathbf{E}\left[S_{t} \mid S_{t-1}\right] \mid S_{t-2}\right] \ldots \mid S_{0}\right] \leqslant(1+\mathbf{E}[R])^{t} \cdot \mathbf{E}[R] .
$$

By applying Markov's inequality, we conclude that

$$
\operatorname{Pr}\left[I_{t} \geqslant n\right] \leqslant \operatorname{Pr}\left[S_{t} \geqslant n\right] \leqslant \frac{(1+\mathbf{E}[R])^{t} \cdot \mathbf{E}[R]}{n} .
$$

Hence $\Omega(\log n)$ rounds are necessary to inform all nodes with probability $1-o(1)$.

\section{Lower Bound for Push-Pull}

Before we present our results about the Push-Pull protocol we show the following general lemma. Recall that $S_{0}=C_{u}$, where $u$ is the single node that is aware of the rumor at the beginning of the protocol. 
Lemma 7.1. Consider the Push-Pull protocol and $\left\{C_{u}: u \in \mathcal{V}\right\}$ be a sequence of positive integers. Then with probability $1-o(1)$, the Push-Pull protocol needs at least

$$
\Omega\left(\frac{\log n-\log S_{0}}{\log \sum_{u \in \mathcal{V}} C_{u}^{2} / n}\right)
$$

rounds to inform all nodes.

Proof. We know the probability that an uninformed node $u$ gets informed by Pull in round $t+1$ is bounded by $I_{t} \cdot C_{u} / n$. Therefore using this bound we have

$$
\begin{aligned}
& \sum_{u \in \mathcal{U}_{t}} \mathbf{E}\left[C_{u} \mathbb{1}(u \text { gets informed by Pull }) \mid S_{t}\right] \\
& =\sum_{u \in \mathcal{U}_{t}} C_{u} \cdot \operatorname{Pr}[u \text { gets informed by Pull in round } t+1] \\
& \leqslant \sum_{u \in \mathcal{U}_{t}} C_{u} \cdot \frac{I_{t} \cdot C_{u}}{n} \leqslant I_{t} \cdot \sum_{u \in \mathcal{V}} \frac{C_{u}^{2}}{n}
\end{aligned}
$$

On the other hand the probability that a node $u \in \mathcal{U}_{t}$ gets informed by Push in round $t+1$ is at most $S_{t} / n$. So we get that

$$
\begin{aligned}
& \sum_{u \in \mathcal{U}_{t}} \mathbf{E}\left[C_{u} \mathbb{1}(u \text { gets informed by Push }) \mid S_{t}\right] \\
& =\sum_{u \in \mathcal{U}_{t}} C_{u} \cdot \operatorname{Pr}[u \text { gets informed by Push in round } t+1] \\
& \leqslant \sum_{u \in \mathcal{U}_{t}} C_{u} \cdot \frac{S_{t}}{n} \leqslant S_{t} \cdot \sum_{u \in \mathcal{V}} \frac{C_{u}^{2}}{n}
\end{aligned}
$$

where the last inequality follows by $C_{u} \leqslant C_{u}^{2}$. Combining the above inequalities implies that

$$
\mathbf{E}\left[S_{t+1} \mid S_{t}\right] \leqslant S_{t}+\left(S_{t}+I_{t}\right) \cdot\left(\sum_{u \in \mathcal{V}} C_{u}^{2} / n\right) \cdot \leqslant\left(1+2 \cdot \sum_{u \in \mathcal{V}} C_{u}^{2} / n\right) \cdot S_{t},
$$

Applying the law of total expectation yields that

$$
\mathbf{E}\left[S_{t}\right]=\mathbf{E}\left[\ldots \mathbf{E}\left[\mathbf{E}\left[S_{t} \mid S_{t-1}\right] \mid S_{t-2}\right] \ldots \mid S_{0}\right] \leqslant\left(1+2 \cdot \sum_{u \in \mathcal{V}} C_{u}^{2} / n\right)^{t} \cdot S_{0}
$$

Using Markov's inequality implies that

$$
\operatorname{Pr}\left[I_{t}=n\right] \leqslant \operatorname{Pr}\left[S_{t}>n / 2\right] \leqslant \frac{\mathbf{E}\left[S_{t}\right]}{n / 2} \leqslant \frac{\left(1+2 \cdot \sum_{u \in \mathcal{V}} C_{u}^{2} / n\right)^{t} \cdot S_{0}}{n / 2},
$$

and the claim follows. 


\section{Push-Pull Protocol with Bounded Mean and Bounded Vari- ance}

This section is devoted to the proof of Theorem 1.3.

Proof. $\left\{C_{u}: u \in \mathcal{V}\right\}$ be a sequence of positive integers each of which is generated independently according to some distribution $R$ with $\mathbf{E}[R]=\mathcal{O}(1)$ and $\operatorname{Var}[R]=\mathcal{O}(1)$. We call $\left\{C_{u}: u \in \mathcal{V}\right\}$ a good sequence if $\sum_{u \in \mathcal{V}} C_{u}^{2}=\mathcal{O}(n)$ and $S_{0}=\mathcal{O}(1)$. Since the origin of the rumor is chosen without knowing $C_{u}, \mathbf{E}\left[S_{0}\right]=\mathbf{E}[R]$. Applying Markov's inequality implies that for any constant $\epsilon>0$ with probability at least $1-\epsilon / 2, S_{0}=\mathcal{O}(1)$. Since $R$ is a probability distribution with bounded variance, $\sum_{u \in \mathcal{V}} \mathbf{E}\left[C_{u}^{2}\right]=\mathcal{O}(n)$. Another application of Markov's inequality implies that with probability $1-\epsilon / 2, \sum_{u \in \mathcal{V}} C_{u}^{2}=\mathcal{O}(n)$. Therefore using a union bound for failure probability of two mentioned events implies that for fixed $\epsilon>0$ with probability at least $1-\epsilon,\left\{C_{u}: u \in \mathcal{V}\right\}$ is a good sequence. Conditioning on the event that $\left\{C_{u}: u \in \mathcal{V}\right\}$ is a good sequence, using Lemma 7.1 implies that with probability at least $1-o(1)$ the Push-Pull protocol needs $\Omega(\log n)$ rounds to inform $n$ nodes and the result follows.

\section{Push-Pull Protocol with Power Law Distribution $2<\beta<3$}

In this section we analyze the Push-Pull protocol where $R$ is a power law distribution with $2<\beta<3$ and show that it only takes $\Theta(\log \log n)$ rounds to inform all with probability $1-o(1)$. To prove the upper bound of $\mathcal{O}(\log \log n)$, we study the protocol in three consecutive phases and show that each phase takes only $\mathcal{O}(\log \log n)$ rounds. After that we show the lower bound $\Omega(\log \log n)$.

\subsection{Proof of the Upper Bound}

\section{The Preliminary Phase.}

This phase starts with just one informed node and ends when $I_{t} \geqslant n^{\frac{1}{\beta-1}} /(2 \cdot \log n)$. Let $T_{1}$ be the first round where $I_{T_{1}} \geqslant n^{\frac{1}{\beta-1}} /(2 \log n)$. We will show that $T_{1}=\mathcal{O}(\log \log n)$. First we claim that $\mathcal{O}(\log \log n)$ rounds are sufficient to have $\log ^{\mathcal{O}(1)} n$ informed nodes. Then we will show that in round $t+1$ with probability $1-e^{-\Omega(\log n)}$ there exists a node $u$ with $C_{u} \geqslant I_{t}^{1+\gamma}, \gamma:=\frac{3-\beta}{2(\beta-2)}>0$, which pulls the rumor and consequently $S_{t+1} \geqslant I_{t}^{1+\gamma}$. Then considering only Push calls it follows that with probability $1-o\left(\frac{1}{\log n}\right)$,

$$
I_{t+2}=I_{t+1}+N_{t+1} \geqslant I_{t+1}+S_{t+1}(1-o(1))>\frac{1}{2} I_{t}^{1+\gamma}
$$

So in every two rounds, $I_{t}$ is increased by a factor of $\frac{1}{2} I_{t}^{\gamma}$ and hence after $\mathcal{O}(\log \log n)$ rounds the phase ends. For a complete proof see the following lemma.

Lemma 9.1. With probability $1-o(1), T_{1}=\mathcal{O}(\log \log n)$. 
Proof. At first we only consider Push calls and apply Lemma 4.1 which states that as long as $S_{t} \leqslant \log ^{\frac{2}{3-\beta}} n$, with probability $1-\mathcal{O}\left(\frac{\log ^{\frac{4}{3-\beta}} n}{n}\right)$,

$$
I_{t+1}=I_{t}+S_{t} \geqslant 2 I_{t} .
$$

Thus as long as $S_{t} \leqslant \log ^{\frac{2}{3-\beta}} n$, in each round the number of informed nodes is at least doubled. So we conclude that with probability $1-o(1), \mathcal{O}(\log \log n)$ rounds are sufficient to inform $\log ^{\frac{2}{3-\beta}} n$ nodes. Let $T_{0}$ be the first round when $I_{T_{0}} \geqslant \log ^{\frac{2}{3-\beta}} n$. Let us define the constant $\gamma:=\frac{3-\beta}{2(\beta-2)}>0$. Let $T$ be the first round such that

$$
I_{T-1}^{(1+\gamma)} \leqslant n^{\frac{1}{\beta-1}} / \log n<I_{T}^{(1+\gamma)} .
$$

Now for any $T_{0} \leqslant t \leqslant T$, we can apply Proposition 3.3 and conclude that with probability $1-o\left(\frac{1}{n}\right)$,

$$
\sum_{u \in \mathcal{L}\left(I_{t}^{1+\gamma}\right)} C_{u} \geqslant L\left(I_{t}^{1+\gamma}\right) \cdot I_{t}^{1+\gamma} \geqslant \frac{n \cdot c_{1} \cdot I_{t}^{(1+\gamma)(2-\beta)}}{2}
$$

So,

$$
\frac{I_{t}}{n} \sum_{u \in \mathcal{L}\left(I_{t}^{1+\gamma}\right)} C_{u} \geqslant \frac{c_{1} \cdot I_{t}^{1+(1+\gamma)(2-\beta)}}{2}=\frac{c_{1} \cdot I_{t}^{3-\beta+\gamma(2-\beta)}}{2} .
$$

We will bound the probability that none of $u \in \mathcal{L}\left(I_{t}^{1+\gamma}\right)$ gets informed by Pull calls in round $t+1$ as follows,

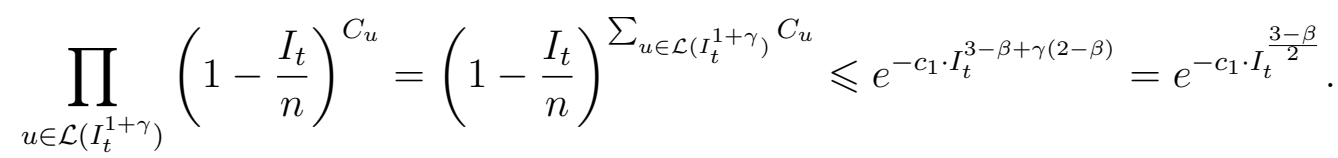

Since for any $t \geqslant T_{0}, I_{t} \geqslant \log \frac{2}{3-\beta} n$, we have that with probability at least $1-n^{-c_{1}}$, at least one node in $\mathcal{L}\left(I_{t}^{1+\gamma}\right)$ gets informed by Pull in round $t+1$. Hence we have that

$$
S_{t+1} \geqslant I_{t}^{1+\gamma}
$$

Let us now consider the Push calls in round $t+2$. By applying Lemma 4.1 we know that as long as $S_{t+1}=o(n)$ with probability $1-o\left(\frac{1}{\log n}\right)$,

$$
S_{t+1}(1-o(1)) \leqslant N_{t+1}
$$

Thus,

$$
I_{t+2} \geqslant I_{t+1}+S_{t+1}(1-o(1))>\frac{I_{t}^{1+\gamma}}{2} .
$$


An inductive argument shows that for any integer $k \geqslant 1$ as long as $I_{T_{0}+2 k-2}^{1+\gamma} \leqslant n^{\frac{1}{\beta-1}} / \log n$, with probability $1-o\left(\frac{k}{\log n}\right)$

$$
I_{T_{0}+2 k}>\left(\frac{1}{2}\right)^{\sum_{i=0}^{k-1}(1+\gamma)^{i}} I_{T_{0}}^{(1+\gamma)^{k}}=\left(\frac{I_{T_{0}}}{2^{\gamma}}\right)^{(1+\gamma)^{k}} \cdot 2^{1 / \gamma}>\left(\frac{\log ^{\frac{2}{3-\beta}} n}{C^{\prime}}\right)^{(1+\gamma)^{k}}
$$

where $C^{\prime}=2^{\gamma}=\mathcal{O}(1)$. So we conclude that after $T_{0}+2 k$ rounds, where $k=o\left(\log _{1+\gamma} \log n\right)$, there are two cases: either $I_{T_{0}+2 k} \geqslant n^{\frac{1}{\beta-1}} /(2 \log n)$ which means $T_{1} \leqslant T_{0}+2 k=\mathcal{O}(\log \log n)$ and we are done, or

$$
I_{T_{0}+2 k}<n^{\frac{1}{\beta-1}} /(2 \log n)<n^{\frac{1}{\beta-1}} / \log n<I_{T_{0}+2 k}^{1+\gamma} .
$$

In the latter case, we change the value $\gamma$ to $\gamma^{\prime}$ which satisfies $I_{T_{0}+2 k}^{1+\gamma^{\prime}}=n^{\frac{1}{\beta-1}} / \log n$ and a similar argument shows that

$$
I_{T_{0}+2 k+2} \geqslant n^{\frac{1}{\beta-1}} /(2 \log n) .
$$

\section{The Middle Phase.}

This phase starts with at least $n^{\frac{1}{\beta-1}} /(2 \log n)$ informed nodes and ends when $I_{t} \geqslant \frac{n}{\log n}$. Let $T_{2}$ be the first round in which $\frac{n}{\log n}$ nodes are informed. We will show that $T_{2}-T_{1}=$ $\mathcal{O}(\log \log n)$. In contrast to the Preliminary Phase where we focus only on an informed node with maximal $C_{u}$, we now consider the number of informed nodes $u$ with a $C_{u}$ above a certain threshold $Z_{t+1}$ which is inversely proportional to $I_{t}$.

Lemma 9.2. Suppose that $I_{t} \geqslant n^{\frac{1}{\beta-1}} /(2 \log n)$ for some round $t$. Then with probability $1-o\left(\frac{1}{n}\right)$,

$$
\left|\mathcal{L}\left(Z_{t+1}\right) \cap \mathcal{I}_{t+1}\right| \geqslant \frac{1}{4} L\left(Z_{t+1}\right)
$$

where $Z_{t+1}:=\frac{n \log \log n}{I_{t}}$.

Proof. We consider two cases. If at least $\frac{1}{4}$ of the nodes in $\mathcal{L}\left(Z_{t+1}\right)$ are already informed (before round $t+1$ ), then the statement of the lemma is true. Otherwise $\left|\mathcal{L}\left(Z_{t+1}\right) \cap \mathcal{U}_{t+1}\right|>$ $\frac{3}{4} L\left(Z_{t+1}\right)$. In the latter case, we define

$$
\mathcal{L}^{\prime}\left(Z_{t+1}\right):=\mathcal{L}\left(Z_{t+1}\right) \cap \mathcal{U}_{t+1} .
$$

Let $X_{u}$ be an indicator random variable for every $u \in \mathcal{L}^{\prime}\left(Z_{t+1}\right)$ so that

$$
X_{u}:= \begin{cases}1 & \text { if } u \text { gets informed by Pull in round } t+1 \\ 0 & \text { otherwise. }\end{cases}
$$


Then we define a random variable $X$ to be $X:=\sum_{u \in \mathcal{L}^{\prime}\left(Z_{t+1}\right)} X_{u}$. Since for every $u \in$ $\mathcal{L}^{\prime}\left(Z_{t+1}\right), C_{u} \geqslant Z_{t+1}=\frac{n \log \log n}{I_{t}}$, it follows that

$$
\operatorname{Pr}\left[X_{u}=1\right]=1-\left(1-\frac{I_{t}}{n}\right)^{C_{u}} \geqslant 1-\left(1-\frac{I_{t}}{n}\right)^{Z_{t+1}}=1-e^{-\Omega(\log \log n)}=1-o(1) .
$$

Thus $\operatorname{Pr}\left[X_{u}=1\right]>\frac{3}{4}$ and $\mathbf{E}[X]=\sum_{u \in \mathcal{L}^{\prime}\left(Z_{t+1}\right)} \operatorname{Pr}\left[X_{u}=1\right]>\frac{3}{4}\left|\mathcal{L}^{\prime}\left(Z_{t+1}\right)\right|$. Since

$$
\left|\mathcal{L}^{\prime}\left(Z_{t+1}\right)\right|=\left|\mathcal{L}\left(Z_{t+1}\right) \cap \mathcal{U}_{t+1}\right|>\frac{3}{4} L\left(Z_{t+1}\right)
$$

it follows that

$$
\mathbf{E}[X] \geqslant \frac{9}{16} L\left(Z_{t+1}\right)
$$

We know that $I_{t} \geqslant n^{\frac{1}{\beta-1}} /(2 \log n)$ and also $I_{t}$ is a non-decreasing function in $t$, so

$$
Z_{t+1}=\frac{n \log \log n}{I_{t}} \leqslant 2 \cdot n^{\frac{\beta-2}{\beta-1}} \log n \log \log n<n^{\frac{1}{\beta-1}} / \log n,
$$

where the last inequality holds because $\beta<3$. Now we can apply Proposition 3.3 (see appendix) to infer that with probability $1-o\left(\frac{1}{n}\right)$,

$$
L\left(Z_{t+1}\right) \geqslant \frac{n \cdot c_{1} \cdot Z_{t+1}^{1-\beta}}{2} \geqslant \frac{c_{1} \cdot \log ^{\beta-1} n}{2}
$$

Therefore,

$$
\mathbf{E}[X] \geqslant \frac{9 \cdot c_{1} \cdot \log ^{\beta-1} n}{32} .
$$

Then applying Theorem 2.1 results into

$$
\operatorname{Pr}\left[X<\frac{\mathbf{E}[X]}{2}\right] \leqslant \operatorname{Pr}\left[|X-\mathbf{E}[X]| \geqslant \frac{\mathbf{E}[X]}{2}\right]<2 e^{-\frac{\mathbf{E}[X]}{10}} \leqslant 2 e^{-\Omega\left(\log ^{\beta-1} n\right)} .
$$

So with probability $1-o\left(\frac{1}{n}\right)$, we have that

$$
\left|\mathcal{L}\left(Z_{t+1}\right) \cap \mathcal{I}_{t+1}\right| \geqslant X \geqslant \frac{\mathbf{E}[X]}{2}>\frac{3\left|\mathcal{L}^{\prime}\left(Z_{t+1}\right)\right|}{8} \geqslant \frac{1}{4} L\left(Z_{t+1}\right),
$$

where the last inequality holds because $\left|\mathcal{L}^{\prime}\left(Z_{t+1}\right)\right|>\frac{3}{4} L\left(Z_{t+1}\right)$.

Lemma 9.3. With probability $1-o(1), T_{2}-T_{1}=\mathcal{O}(\log \log n)$.

Proof. Since $I_{t} \geqslant n^{\frac{1}{\beta-1}} /(2 \log n), Z_{t+1}=\frac{n \log \log n}{I_{t}}<n^{\frac{1}{\beta-1}} / \log n$, using Proposition 9.2 results into a lower bound for $\left|\mathcal{L}\left(Z_{t+1}\right) \cap \mathcal{I}_{t+1}\right|$. So with probability $1-o\left(\frac{1}{n}\right)$,

$$
S_{t+1}=\sum_{u \in I_{t+1}} C_{u} \geqslant\left|\mathcal{L}\left(Z_{t+1} \cap \mathcal{I}_{t+1}\right)\right| \cdot Z_{t+1} \geqslant \frac{1}{4} L\left(Z_{t+1}\right) \cdot Z_{t+1}
$$


By applying Proposition 3.3, we conclude that with probability $1-o\left(\frac{1}{n}\right), L\left(Z_{t+1}\right) \geqslant$ $\frac{n \cdot c_{1} \cdot Z_{t+1}^{1-\beta}}{2}$. Therefore, with probability $1-o\left(\frac{1}{n}\right)$,

$$
S_{t+1} \geqslant \frac{n \cdot c_{1} \cdot Z_{t+1}^{2-\beta}}{8}
$$

As long as $S_{t+1}=o(n)$, we can apply Lemma 4.2 for the Push protocol to round $t+2$ implying that with probability $1-o\left(\frac{1}{\log n}\right)$,

$$
I_{t+2}=I_{t+1}+N_{t} \geqslant I_{t+1}+S_{t+1}(1-o(1))
$$

Thus,

$$
I_{t+2}>\frac{S_{t+1}}{2} \geqslant \frac{c_{1}}{16} n \cdot Z_{t+1}^{2-\beta}=\frac{c_{1}}{16} \cdot n^{3-\beta} \cdot \log \log ^{2-\beta} n \cdot I_{t}^{\beta-2} .
$$

By an inductive argument, we obtain that for any integer $k \geqslant 1$ with $S_{t+k}=o(n)$, it holds with probability $1-o\left(\frac{k}{\log n}\right)$,

$$
I_{t+2 k}>\left(\frac{c}{16} n^{3-\beta} \cdot \log \log ^{2-\beta} n\right)^{\sum_{i=0}^{k-1}(\beta-2)^{i}} I_{t}^{(\beta-2)^{k}}=\left(\frac{c}{16} n^{3-\beta} \cdot \log \log ^{2-\beta} n\right)^{\frac{1-(\beta-2)^{k}}{3-\beta}} I_{t}^{(\beta-2)^{k}} .
$$

Therefore there exists $k=\mathcal{O}\left(\log _{\frac{1}{\beta-2}} \log n\right)$ such that

$$
\begin{aligned}
I_{t+2 k} & \geqslant\left(\frac{c}{16} n^{3-\beta} \cdot \log \log ^{2-\beta} n\right)^{\frac{1-\mathcal{O}(1 / \log n)}{3-\beta}} I_{t}^{1 / \log n} \\
& =\Omega\left(n^{1-\mathcal{O}(1 / \log n)}\left(\frac{c}{16} \cdot \log \log ^{2-\beta} n\right)^{\frac{1-\mathcal{O}(1 / \log n)}{3-\beta}}\right)=\Omega\left(\frac{n}{\log \log ^{\delta} n}\right),
\end{aligned}
$$

where $\delta=\frac{\beta-2}{3-\beta}(1-\mathcal{O}(1 / \log n))>0$. Hence $T_{2} \leqslant T_{1}+2 k=T_{1}+\mathcal{O}(\log \log n)$ with probability $1-o(1)$.

\section{The Final Phase.}

This phase starts with at least $\frac{n}{\log n}$ informed nodes. Since the runtime of our Push-Pull protocol is stochastically smaller than the runtime of the standard Push-Pull protocol (i.e. $C_{u}=1$ for every $u \in V$ ), we simply use the result by Karp et. al in [24, Theorem 2.1] for the standard Push-Pull protocol which states that once $I_{t} \geqslant \frac{n}{\log n}$, additional $\mathcal{O}(\log \log n)$ rounds are with probability $1-o(1)$ sufficient to inform all $n$ nodes.

\subsection{Proof of the Lower Bound}

Since increasing the number of informed nodes can only decrease the runtime of the

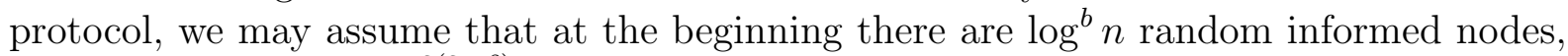
where $b:=\max \left\{4,2+\frac{3(3-\beta)}{\beta-2}\right\}$. Applying Markov's inequality to the random variable $S_{0}$ 
implies that with probability $1-o\left(\frac{1}{\log n}\right), \log ^{b} n \leqslant S_{0} \leqslant \log ^{2+b} n$. In the following we lower bound the number of rounds to reach $n^{\frac{1}{\log \log n}}$ informed nodes. We do this by keeping track of the largest value of $C_{u}$ among all informed nodes and show that this value does not exceed $I_{t}^{\frac{1}{\beta-2}} \log \frac{3}{\beta-2} n$ with high probability.

By Fact 3.2, with probability $1-o\left(\frac{1}{\log n}\right)$ we have $\max _{u \in \mathcal{V}} C_{u} \leqslant n^{\frac{1}{\beta-1}} \log n$. Let $i^{*}$ be the smallest positive integer so that $2^{i^{*}} \geqslant n^{\frac{1}{\beta-1}} / \log n$. Then $i^{*}<\log n$. Let us define the set $\mathcal{M}_{i}:=\left\{u \in \mathcal{V}: 2^{i-1} \leqslant C_{u}<2^{i}\right\}$ for $1 \leqslant i \leqslant i^{*}-1$ and $\mathcal{M}_{i^{*}}:=\left\{u \in \mathcal{V}: 2^{i^{*}-1} \leqslant\right.$ $\left.C_{u} \leqslant n^{\frac{1}{\beta-1}} \log n\right\}$. We denote the size of $\mathcal{M}_{i}$ with $M_{i}$. By definition, for any $1 \leqslant i \leqslant i^{*}$, $M_{i} \leqslant L\left(2^{i-1}\right)$. Applying Proposition 3.3 implies that with probability $1-o\left(\frac{1}{n}\right)$ for any $1 \leqslant i \leqslant i^{*}$ we have $M_{i} \leqslant \frac{3}{2} \cdot c_{2} \cdot n \cdot 2^{(i-1)(1-\beta)}$. Let us define the indicator random variable $Z_{u}^{i}$ for every $u \in \mathcal{U}_{t} \cap \mathcal{M}_{i}$ as follows:

$$
Z_{u}^{i}:= \begin{cases}1 & \text { if } u \text { gets informed by Pull in round } \mathrm{t}+1, \\ 0 & \text { otherwise. }\end{cases}
$$

Hence, $\operatorname{Pr}\left[Z_{u}^{i}=1\right] \leqslant C_{u} \cdot \frac{I_{t}}{n} \leqslant \frac{I_{t} \cdot 2^{i}}{n}$. Let $P_{i}$ be the probability that at least one node in $\mathcal{U}_{t} \cap \mathcal{M}_{i}$ gets informed by Pull in round $t+1$. Then, for any $1 \leqslant i \leqslant i^{*}-1$,

$$
P_{i} \leqslant \sum_{u \in \mathcal{U}_{t} \cap \mathcal{M}_{i}} \operatorname{Pr}\left[Z_{u}^{i}=1\right] \leqslant M_{i} \cdot \frac{I_{t}}{n} \cdot 2^{i} \leqslant 3 \cdot c_{2} \cdot I_{t} \cdot 2^{(i-1)(2-\beta)} .
$$

Since $2^{i^{*}} \geqslant n^{\frac{1}{\beta-1}} / \log n$ and $C_{u} \leqslant n^{\frac{1}{\beta-1}} \log n$ with probability $1-o\left(\frac{1}{\log n}\right)$,

$$
\begin{aligned}
P_{i^{*}} \leqslant \sum_{u \in \mathcal{U}_{t} \cap \mathcal{M}_{i^{*}}} \operatorname{Pr}\left[Z_{u}^{i}=1\right] & \leqslant \frac{3}{2} \cdot c_{2} \cdot n \cdot 2^{\left(i^{*}-1\right)(1-\beta)} \cdot \frac{I_{t}}{n} \cdot n^{\frac{1}{\beta-1}} \log n \\
& \leqslant 6 \cdot c_{2} \cdot I_{t} \cdot n^{\frac{2-\beta}{\beta-1}} \log ^{\beta-1} \cdot n .
\end{aligned}
$$

So as long as $I_{t} \leqslant n^{\frac{1}{\log \log n}}, P_{i^{*}}=o\left(\frac{1}{\log ^{3} n}\right)$. We define $\Delta_{t}:=S_{t}^{\frac{1}{\beta-2}} \log ^{\frac{3}{\beta-2}} n$. Let $1 \leqslant i_{t} \leqslant i^{*}$ be the smallest integer so that $2^{i_{t}} \geqslant \Delta_{t}$. Then for any $i_{t} \leqslant i \leqslant i^{*}$ we have,

$$
P_{i} \leqslant 3 \cdot c_{2} \cdot 2^{\beta-2} \cdot I_{t} \cdot 2^{i(2-\beta)} \leqslant 6 \cdot c_{2} \cdot I_{t} \cdot \Delta_{t}^{2-\beta} \leqslant 6 \cdot c_{2} \cdot \log ^{-3} n .
$$

Let $E_{t}$ be the event that no node with $C_{u} \geqslant \Delta_{t}$ gets informed by Pull in round $t+1$. Then we have

$$
\operatorname{Pr}\left[E_{t}\right] \geqslant 1-\sum_{i=i_{t}}^{i^{*}} P_{i} \geqslant 1-o\left(\frac{1}{\log n}\right) .
$$

Let us define $S_{t+1}^{(1)}:=\sum_{u \in \mathcal{N}_{t}^{\text {pull }}} C_{u}$. Conditioning on the event $E_{t}$ we obtain that

$$
\begin{aligned}
\mathbf{E}\left[S_{t+1}^{(1)} \mid S_{t}\right] & \leqslant \sum_{i=1}^{i_{t}} \sum_{u \in \mathcal{U}_{t} \cap \mathcal{M}_{i}} C_{u} \cdot \frac{\operatorname{Pr}\left[Z_{u}^{i}=1\right]}{\operatorname{Pr}\left[E_{t}\right]} \\
& \leqslant(1+o(1)) \cdot \sum_{i=1}^{i_{t}} 2^{i} \cdot M_{i} \cdot \frac{I_{t}}{n} \cdot 2^{i} \leqslant(1+o(1)) \cdot \frac{S_{t}}{n} \cdot \sum_{i=1}^{i_{t}} 2^{i} \cdot M_{i} \cdot 2^{i} .
\end{aligned}
$$


By definition of $i_{t}$, we have $2^{i_{t}} \leqslant 2 \cdot \Delta_{t}$ and also we have $M_{i} \leqslant L\left(2^{i-1}\right) \leqslant \frac{3}{2} \cdot c_{2} \cdot 2^{(i-1)(1-\beta)} \cdot n$. Hence the last sum is bounded by

$$
\begin{aligned}
(1+o(1)) \cdot \sum_{i=1}^{i_{t}} 2^{2 i} \cdot I_{t} \cdot 2^{(i-1)(1-\beta)} \leqslant 24 \cdot c_{2} \cdot I_{t} \cdot 2^{i_{t}(3-\beta)} & \leqslant 24 \cdot c_{2} \cdot I_{t} \cdot\left(2 \cdot \Delta_{t}\right)^{3-\beta} \\
& \leqslant 24 \cdot c_{2} \cdot S_{t}^{1+\frac{3-\beta}{\beta-2}} \log ^{\frac{3(3-\beta)}{\beta-2}} n .
\end{aligned}
$$

Conditioning on the event $E_{t}$ and applying Markov's inequality imply that with probability $1-o\left(\frac{1}{\log n}\right)$,

$$
S_{t+1}^{(1)} \leqslant \log ^{2} n \cdot \mathbf{E}\left[S_{t+1}^{(1)} \mid S_{t}\right] \leqslant 24 \cdot c_{2} \cdot S_{t}^{1+\frac{3-\beta}{\beta-2}} \log ^{2+\frac{3(3-\beta)}{\beta-2}} n .
$$

Let us define the indicator random variable $Y_{u}$ for every $u \in \mathcal{U}_{t}$ as follows:

$$
Y_{u}:= \begin{cases}1 & \text { if } u \text { gets informed by Push in round } \mathrm{t}+1, \\ 0 & \text { otherwise. }\end{cases}
$$

Then we have $\operatorname{Pr}\left[Y_{u}=1\right] \leqslant S_{t} / n$. Let $A$ denote the event that $\sum_{u \in \mathcal{V}} C_{u} \leqslant n \cdot \log ^{2} n$. Since $\mathbf{E}[R]=\mathcal{O}(1)$, applying Markov's inequality implies that $\operatorname{Pr}[A] \geqslant 1-o(1 / \log n)$. Let us define $S_{t+1}^{(2)}:=\sum_{u \in \mathcal{N}_{t}^{\text {Push }}} C_{u}$. Conditioning on the event $A$ we have

$$
\mathbf{E}\left[S_{t+1}^{(2)} \mid S_{t}\right]=\sum_{u \in \mathcal{U}_{t}} C_{u} \cdot \frac{\operatorname{Pr}\left[Y_{u}=1\right]}{\operatorname{Pr}[A]} \leqslant(1+o(1)) \cdot \sum_{u \in \mathcal{V}} C_{u} \cdot \frac{S_{t}}{n} \leqslant(1+o(1)) \cdot S_{t} \cdot \log ^{2} n .
$$

Conditioning on $A$ and applying Markov's inequality implies that with probability 1 $o(1 / \log n)$,

$$
S_{t+1}^{(2)} \leqslant \log ^{2} n \cdot \mathbf{E}\left[S_{t+1}^{(2)} \mid S_{t}\right] \leqslant S_{t} \cdot \log ^{4} n
$$

Combining inequalities (18) and (19) implies that with probability $1-o(1 / \log n)$ for every $0 \leqslant t \leqslant \log \log n$

$$
\begin{aligned}
S_{t+1} \leqslant S_{t}+S_{t+1}^{(1)}+S_{t+1}^{(2)} & \leqslant S_{t}+24 \cdot c_{2} \cdot S_{t}^{1+\frac{3-\beta}{\beta-2}} \log ^{2+\frac{3(3-\beta)}{\beta-2}} n+S_{t} \cdot \log ^{4} n \\
& \leqslant S_{t}+24 \cdot c_{2} \cdot S_{t}^{b+1}+S_{t}^{2} \leqslant S_{t}^{b+2}
\end{aligned}
$$

where the last inequality holds because $b=\max \left\{4,2+\frac{3(3-\beta)}{\beta-2}\right\}$ and $\log ^{b} n \leqslant I_{t} \leqslant S_{t}$. We know that with probability $1-o(1 / \log n)$ we have $S_{0} \leqslant \log ^{b+2} n$. An inductive argument shows that for every $1 \leqslant t \leqslant \log \log n$ with probability $1-o(1), S_{t} \leqslant S_{0}^{(b+2)^{t}} \leqslant \log ^{(b+2)^{t+1}}$. If we set $T:=\frac{1}{2} \cdot \log _{b+2} \log n$, then with probability $1-o(1)$ we have $S_{T}<n^{1 / \log \log n}$. Thus $T=\Omega(\log \log n)$ rounds are necessary to inform all nodes with probability $1-o(1)$. This finishes the proof of the lower bound of $\Omega(\log \log n)$. 


\section{Push-Pull Protocol with Power Law Distribution $\beta=3$}

In this section we analyse the Push-Pull protocol where $R$ is a power law distribution with $\beta=3$ and show that the Push-Pull protocol takes $\Theta\left(\frac{\log n}{\log \log n}\right)$ rounds to inform all $n$ nodes. Throughout this section we assume that the power law distribution with $\beta=3$ has an additional property in which for every positive integer $z$

$$
\operatorname{Pr}[R=z] \geqslant c \cdot z^{-3},
$$

where $c>0$ is fixed. Let us define $\mathcal{L}^{*}(z)=\left\{u: C_{u}=z\right\}$ and $L^{*}(z)=\left|\mathcal{L}^{*}(z)\right|$. Also we define $\mathcal{I}_{t}(z)=\mathcal{I}_{t} \cap \mathcal{L}^{*}(z)$ and $\mathcal{N}_{t}(z)=\mathcal{N}_{t} \cap \mathcal{L}^{*}(z)$, whose sizes are denoted by $I_{t}(z)$ and $N_{t}(z)$ respectively. $N_{t}^{\text {Push }}(z)$ and $N_{t}^{\text {Pull }}(z)$ are denoted the size of the newly informed nodes with $C_{u}=z$ by Push and Pull transmissions respectively. In the following we show a useful fact about the $L^{*}(z)$.

Fact 10.1. Let $R$ be a power law distribution with $\beta=3$. Then for every $z=\mathcal{O}\left(n^{1 / 4}\right)$, with probability $1-o\left(\frac{1}{n}\right)$ we have that

$$
\frac{n \cdot \operatorname{Pr}[R=z]}{2} \leqslant L^{*}(z) \leqslant \frac{3 \cdot n \cdot \operatorname{Pr}[R=z]}{2} .
$$

Proof. We know that $\mathbf{E}\left[L^{*}(z)\right]=n \cdot \operatorname{Pr}[R=z]$. By using the inequality (20) we have that for any $z=\mathcal{O}\left(n^{1 / 4}\right), \operatorname{Pr}[R=z]=\Omega\left(n^{-3 / 4}\right)$. Then we have that $\mathbf{E}\left[L^{*}(z)\right]=\Omega\left(n^{2 / 5}\right)$ and using a Chernoff bound 2.1 yields that with probability $1-o\left(\frac{1}{n}\right)$ the inequality in the statement holds.

\subsection{Proof of Lower Bound}

Theorem 10.2. With probability $1-o(1)$, the Push-Pull needs at least $\Omega\left(\frac{\log n}{\log \log n}\right)$ rounds to inform all $n$ nodes.

Proof. Let $\left\{C_{u}: u \in \mathcal{V}\right\}$ be a sequence of positive integers where every $C_{u}$ is generated independently according to a power law distribution with $\beta=3$. We call a sequence $\left\{C_{u}: u \in \mathcal{V}\right\}$ is good if it fulfills three conditions:

1. For every $u \in \mathcal{V}, C_{u}<n$.

2. $S_{0}=\mathcal{O}(\log n)$.

3. $\sum_{u \in \mathcal{V}} \frac{C_{u}^{2}}{n}=\mathcal{O}\left(\log ^{2} n\right)$.

In the following we show that with probability $1-o(1)$ every sequence $\left\{C_{u}, u \in \mathcal{V}\right\}$ is good. By definition of power law distribution for $\beta=3$ we have that

$$
\operatorname{Pr}\left[C_{u} \leqslant n\right]>1-\frac{c_{1}}{n^{2}}=1-o(1) .
$$


We know that $\mathbf{E}[R]=\mathcal{O}(1)$, so Markov'e inequality implies that with probability 1 $\mathcal{O}\left(\frac{1}{\log n}\right), S_{0}=\mathcal{O}(\log n)$. Conditioning on the event that for every $u \in \mathcal{V}, C_{u}<n$ we get

$$
\mathbf{E}\left[C_{u}^{2} \mid C_{u} \leqslant n\right] \leqslant \frac{\sum_{z=1}^{n} \operatorname{Pr}\left[R^{2} \geqslant z\right]}{\operatorname{Pr}\left[C_{u} \leqslant n\right]} \leqslant(1+o(1)) \cdot c_{1} \sum_{z=1}^{n} \frac{1}{z}=(1+o(1)) \cdot c_{1} \cdot \log n .
$$

So applying Markov's inequality yields that with probability $1-\mathcal{O}\left(\frac{1}{\log n}\right)$,

$$
\sum_{u \in \mathcal{V}} \frac{C_{u}^{2}}{n}=\mathcal{O}\left(\log ^{2} n\right) .
$$

Therefore we have that with probability $1-o(1)$, the sequence $\left\{C_{u}: u \in \mathcal{V}\right\}$ is good. Conditioning on this event and then applying Lemma 7.1 shows that with probability $1-o(1)$ the Push-Pull needs at least

$$
\Omega\left(\frac{\log n-\log S_{0}}{\log \sum_{u \in \mathcal{V}} C_{u}^{2} / n}\right)=\Omega\left(\frac{\log n}{\log \log n}\right)
$$

rounds to inform $n$ nodes.

\subsection{Proof of Upper Bound}

Before we present a proof for the upper bound we show following two lemmas.

Lemma 10.3. Suppose that $S_{t} \leqslant \frac{n}{\log ^{6} n}$ and $z \leqslant \min \left\{\frac{n}{I_{t} \cdot \log ^{6} n}, \mathcal{O}\left(n^{\frac{1}{4}}\right)\right\}$. Then with probability $1-o\left(\frac{1}{\log n}\right)$, for any round $t=\mathcal{O}(\log n)$ we have that

$$
\left|\mathcal{U}_{t}(z) \cap \mathcal{L}^{*}(z)\right| \geqslant \frac{L^{*}(z)}{2} \geqslant \frac{n \cdot \operatorname{Pr}[R=z]}{4} .
$$

Proof. By considering the Push call we have that the size of newly informed nodes is bounded by $S_{t}$. Since they are chosen randomly, we have that

$$
\mathbf{E}\left[N_{t}^{\text {Push }}(z) \mid S_{t}\right] \leqslant S_{t} \cdot \operatorname{Pr}[R=z] .
$$

On the other hand we have that

$\mathbf{E}\left[N_{t}^{\text {Pull }}(z) \mid I_{t}\right] \leqslant \sum_{u \in \mathcal{U}_{t} \cap \mathcal{L}^{*}(z)} \operatorname{Pr}[u$ gets informed by Pull in round $t+1]$

$\leqslant L^{*}(z) \cdot \operatorname{Pr}[u$ gets informed by Pull in round $t+1], \quad$ since $\left|\mathcal{U}_{t} \cap \mathcal{L}^{*}(z)\right| \leqslant L^{*}(z)$.

$$
=L^{*}(z) \cdot\left(1-\left(1-\frac{I_{t}}{n}\right)^{z}\right) \leqslant L^{*}(z) \cdot \frac{2 \cdot I_{t} \cdot z}{n}
$$

where the last inequality holds because we assume that $\frac{I_{t}}{n} \leqslant \frac{S_{t}}{n}<\frac{1}{2}$ and for any $0 \leqslant$ $a \leqslant \frac{\log 2}{2}, \mathrm{e}^{-2 a} \leqslant 1-a \leqslant e^{-a}$. Applying Fact 10.1 shows that for any $z=\mathcal{O}\left(n^{\frac{1}{4}}\right)$ with probability $1-o\left(\frac{1}{n}\right)$ we have

$$
\frac{n \cdot \operatorname{Pr}[R=z]}{2} \leqslant L^{*}(z) \leqslant \frac{3 \cdot n \cdot \operatorname{Pr}[R=z]}{2} .
$$


Thus,

$$
\mathbf{E}\left[N_{t}^{\text {Pull }}(z) \mid S_{t}\right] \leqslant 3 \cdot I_{t} \cdot z \cdot \operatorname{Pr}[R=z] .
$$

Combining (21) and (23) implies that

$$
\mathbf{E}\left[N_{t}(z) \mid S_{t}, I_{t}\right] \leqslant S_{t} \cdot \operatorname{Pr}[R=z]+3 \cdot I_{t} \cdot z \cdot \operatorname{Pr}[R=z]
$$

We know that $I_{t+1}(z)=I_{0}(z)+\sum_{i=1}^{t} N_{i}(z)$. Using the linearity of expectation we have that

$$
\begin{aligned}
\mathbf{E}\left[I_{t+1}(z) \mid S_{i}, I_{i}, 0 \leqslant i \leqslant t\right] & =I_{0}(z)+\sum_{i=0}^{t} \mathbf{E}\left[N_{i}(z) \mid S_{i}, I_{i}\right] \\
& \leqslant I_{0}(z)+\operatorname{Pr}[R=z] \cdot \sum_{i=0}^{t}\left(S_{i}+3 \cdot I_{i} \cdot z\right) \\
& \leqslant 1+\operatorname{Pr}[R=z] \cdot(t+1) \cdot\left(S_{t}+z \cdot 3 \cdot I_{t}\right),
\end{aligned}
$$

where the last inequality comes from the fact that $S_{i}$ and $I_{i}$ are non-decreasing function in $t$. By assumption $z \leqslant \min \left\{\frac{n}{I_{t} \cdot \log ^{6} n}, \mathcal{O}\left(n^{\frac{1}{4}}\right)\right\}$ and $S_{t} \leqslant \frac{n}{\log ^{6} n}$, for any round $t=\mathcal{O}(\log n)$ we have that

$$
\mathbf{E}\left[I_{t+1}(z) \mid S_{i}, I_{i}, 1 \leqslant i \leqslant t\right] \leqslant 2 \cdot(t+1) \cdot\left(S_{t}+3 \cdot I_{t} \cdot z\right) \cdot \operatorname{Pr}[R=z] \leqslant \frac{n \cdot \operatorname{Pr}[R=z]}{\log ^{4} n} .
$$

Applying Markov's inequality shows that with probability $1-o\left(\frac{1}{\log n}\right)$ for any round $t=$ $\mathcal{O}(\log n)$

$$
I_{t+1}(z) \leqslant \log ^{2} n \cdot \mathbf{E}\left[I_{t+1}(z) \mid S_{i}, I_{i}, 0 \leqslant i \leqslant t\right] \leqslant \frac{n \cdot \operatorname{Pr}[R=z]}{\log ^{2} n} \leqslant \frac{L^{*}(z)}{2},
$$

where the last inequality follows from inequality $(22)$. Therefore we infer that with probability $1-o\left(\frac{1}{\log n}\right),\left|\mathcal{U}_{t}(z) \cap \mathcal{L}^{*}(z)\right| \geqslant \frac{L^{*}(z)}{2}$.

Lemma 10.4. Suppose that $I_{t}=\mathrm{e}^{\Omega\left(\frac{\log n}{\log \log n}\right)}$ and $S_{t} \leqslant \frac{n}{\log ^{6} n}$. Then with probability $1-o(1)$, the Push-Pull protocol needs $\mathcal{O}\left(\frac{\log n}{\log \log n}\right)$ rounds to inform at least $\mathrm{e}^{\log n-\frac{\log n}{\log \log n}}$ nodes.

Proof. Let $X_{u}$ be an indicator random variable for every $u \in \mathcal{U}_{t}(z) \cap \mathcal{L}^{*}(z)$ so that

$$
X_{u}:= \begin{cases}1 & \text { if } u \text { gets informed by Pull in round } t+1 \\ 0 & \text { otherwise. }\end{cases}
$$

Then we define the random variable $X_{t}(z):=\sum_{u \in \mathcal{U}_{t}(z) \cap \mathcal{L}^{*}(z)} X_{u}$. Let us define $z_{t}=$ $\min \left\{I_{t}^{1 / 4}, \frac{n}{I_{t} \cdot \log ^{6} n}\right\}$. Using the approximation $\mathrm{e}^{-2 \cdot a} \leqslant 1-a \leqslant \mathrm{e}^{-a}, 0 \leqslant a \leqslant 1 / 2$, results that for any $z \leqslant z_{t}$ we have

$$
\operatorname{Pr}\left[X_{u}=1\right]=1-\left(1-\frac{I_{t}}{n}\right)^{z} \geqslant 1-\mathrm{e}^{-\frac{I_{t} \cdot z}{n}} \geqslant \frac{I_{t} \cdot z}{2 \cdot n},
$$


Applying Lemma 10.3 shows that with probability $1-o\left(\frac{1}{\log n}\right)$ for any $z \leqslant z_{t}$ and any round $t=\mathcal{O}(\log n)$,

$$
\mathbf{E}\left[X_{t}(z)\right]=\sum_{u \in \mathcal{U}_{t}(z) \cap \mathcal{L}^{*}(z)} \operatorname{Pr}\left[X_{u}=1\right]>\frac{L^{*}(z) \cdot I_{t} \cdot z}{4 \cdot n} \geqslant \frac{I_{t} \cdot z \cdot \operatorname{Pr}[R=z]}{8} \geqslant \frac{c \cdot I_{t}}{I_{t}^{\frac{3}{4}}}
$$

where the last inequality holds because $\operatorname{Pr}[R=z] \geqslant \frac{c}{z^{3}}$. Since $I_{t}=e^{\Omega\left(\frac{\log n}{\log \log n}\right)}$ and $X_{u}$ 's are independent, applying a Chernoff bound 2.1 implies that with probability $1-o\left(\frac{1}{n}\right)$,

$$
X_{t}(z) \geqslant \frac{\mathbf{E}\left[X_{t}(z)\right]}{2}
$$

Using the above inequality and inequality $(24)$ shows that that with probability $1-o\left(\frac{1}{\log n}\right)$ there exists a constant $C$ so that

$$
S_{t+1} \geqslant \sum_{z=1}^{z_{t}} X_{t}(z) \cdot z \geqslant \frac{I_{t}}{16} \sum_{z=1}^{z_{t}} z^{2} \cdot \operatorname{Pr}[R=z] \geqslant \frac{c \cdot I_{t}}{16} \sum_{z=1}^{z_{t}} \frac{1}{z}=I_{t} \cdot C \cdot \log z_{t} .
$$

For any positive integer $k$ in which $I_{t+k} \in\left[\mathrm{e}^{\Omega\left(\frac{\log n}{\log \log n}\right)}, \mathrm{e}^{\log n-\frac{\log n}{\log \log n}}\right]$, we have that

$$
\mathrm{e}^{\Omega\left(\frac{\log n}{\log \log n}\right)} \leqslant z_{t}
$$

Hence from the above inequality we conclude that here exists a constant $C_{1}$ so that

$$
S_{t+1} \geqslant C_{1} \cdot I_{t} \cdot \frac{\log n}{\log \log n} \geqslant C_{1} \cdot I_{t} \cdot \sqrt{\log n}
$$

Considering only Push transmission for $S_{t}=o(n)$ and applying Lemma 4.2 implies that with probability $1-o\left(\frac{1}{\log n}\right)$

$$
I_{t+2} \geqslant \frac{S_{t+1}}{2} \geqslant \frac{C_{1} \cdot I_{t} \cdot \sqrt{\log n}}{2}
$$

An inductive argument shows that for any integer $k$ as long as $S_{t+2 k}=\frac{n}{\log ^{6} n}$ with probability $1-o(1)$,

$$
I_{t+2 k} \geqslant I_{t} \cdot\left(\frac{C_{1} \cdot \sqrt{\log n}}{2}\right)^{k} .
$$

Thus there is a $k=\mathcal{O}\left(\frac{\log n}{\log \log n}\right)$ so that after $t+2 k$ rounds there are at least $\mathrm{e}^{\log n-\frac{\log n}{\log \log n}}$ informed nodes.

Corollary 10.5. let $R$ be a power law distribution with $\beta=3$. Then with probability $1-o(1)$, the Push-Pull protocol in informs all $n$ nodes in $\mathcal{O}\left(\frac{\log n}{\log \log n}\right)$ rounds. 
Proof. Applying Corollary 4.4 results that as long as $S_{t}=o(n)$ with probability $1-o(1)$, for any round $t=\mathcal{O}(\log n)$,

$$
I_{t} \geqslant\left(\frac{3}{2}\right)^{t} \cdot I_{0}
$$

So after $\mathcal{O}\left(\frac{\log n}{\log \log n}\right)$ rounds there are at least $\mathrm{e}^{\Omega\left(\frac{\log n}{\log \log n}\right)}$ informed nodes. Now we apply Lemma 10.4 and conclude that after $\mathcal{O}\left(\frac{\log n}{\log \log n}\right)$ rounds we have at least $\mathrm{e}^{\log n-\frac{\log n}{\log \log n}}$ informed nodes. Another application of Corrolarry 4.4 implies that after $\mathcal{O}\left(\frac{\log n}{\log \log n}\right)$ rounds we have at least $\frac{n}{\log \log n}$ informed nodes. Since we have enough number of informed nodes using the result by Karp et. al in [24, Theorem 2.1] for the standard Push-Pull protocol shows that once $I_{t} \geqslant \frac{n}{\log n}$, with probability $1-o(1)$ additional $\mathcal{O}(\log \log n)$ rounds are sufficient to inform all $n$ nodes.

\section{Generating a New $C_{u}^{t}$ in Each Round (Theorem 1.6)}

In this section we analaysis the Push-Pull protocol for a new model. In this model according to some distribution $R$, at the beginning of each round $t$, every node $u$ generates a random natural number $C_{u}^{t} \geqslant 1$ independent of all other nodes. Then in round $t$, the Push-Pull protocol disseminates the information according to $\left\{C_{u}^{t}: u \in \mathcal{V}\right\}$, i.e., node $u$ calls $C_{u}^{t}$ random nodes. In the following we show that if we have $\mathbf{E}[R]=\mathcal{O}(1)$. Then with probability $1-o(1)$, the Push-Pull protocol needs $\Omega(\log n)$ rounds to inform all nodes.

Proof. The probability that a node $u \in \mathcal{U}_{t}$ gets informed by Pull is as follows:

$$
\begin{aligned}
& \operatorname{Pr}[u \text { gets informed by Pull in round } t+1] \\
= & \sum_{x=1}^{\infty} \operatorname{Pr}\left[u \text { gets informed by Pull in round } t+1 \mid R_{u}^{t+1}=x\right] \cdot \operatorname{Pr}\left[R_{u}^{t+1}=x\right] \\
= & \sum_{x=1}^{\left\lfloor\frac{n}{2 I_{t}}\right\rfloor}\left(1-\left(1-\frac{I_{t}}{n}\right)^{x}\right) \cdot \operatorname{Pr}\left[R_{u}^{t+1}=x\right]+\sum_{x=\left\lfloor\frac{n}{2 I_{t}}\right\rfloor+1}^{\infty}\left(1-\left(1-\frac{I_{t}}{n}\right)^{x}\right) \cdot \operatorname{Pr}\left[R_{u}^{t+1}=x\right] \\
\leqslant & \frac{I_{t}}{n} \sum_{x=1}^{\left\lfloor\frac{n}{2 I_{t}}\right\rfloor} x \cdot \operatorname{Pr}\left[R_{u}^{t+1}=x\right]+\sum_{x=\left\lfloor\frac{n}{2 I_{t}}\right\rfloor+1}^{\infty} \operatorname{Pr}\left[R_{u}^{t+1}=x\right] \quad\left(\text { since } 1-\left(1-\frac{I_{t}}{n}\right)^{x} \leqslant \frac{I_{t} \cdot x}{n}\right) \\
\leqslant & \frac{I_{t}}{n} \cdot \mathbf{E}[R]+\operatorname{Pr}\left[R_{u}^{t+1}>\left\lfloor\frac{n}{2 I_{t}}\right\rfloor\right] \leqslant \frac{I_{t}}{n} \cdot \mathbf{E}[R]+\frac{2 I_{t}}{n} \cdot \mathbf{E}[R],
\end{aligned}
$$

where the last inequality is due to Markov's inequality. Recall that $N_{t}^{\text {Pull }}$ and $N_{t}^{\text {Push }}$ are the number of newly informed nodes by Pull and Push calls in round $t+1$ respectively. 
Hence,

$$
\begin{aligned}
\mathbf{E}\left[N_{t}^{\text {Pull }} \mid I_{t}\right] & =\sum_{u \in \mathcal{U}_{t}} \operatorname{Pr}[u \text { gets informed by Pull in round } t+1] \\
& \leqslant \frac{U_{t} \cdot I_{t} \cdot 3 \cdot \mathbf{E}[R]}{n}<3 \cdot I_{t} \cdot \mathbf{E}[R]
\end{aligned}
$$

Recall that $S_{t}$ is the number of Push calls by informed nodes in round $t+1$. Therefore, $N_{t}^{\text {Push }} \leqslant S_{t}$ and

$$
\mathbf{E}\left[N_{t}^{\text {Push }} \mid I_{t}\right] \leqslant \mathbf{E}\left[S_{t} \mid I_{t}\right]=\sum_{u \in I_{t}} \mathbf{E}\left[C_{u}^{t+1}\right]=I_{t} \cdot \mathbf{E}[R]
$$

Hence,

$$
\mathbf{E}\left[I_{t+1} \mid I_{t}\right] \leqslant I_{t}+\mathbf{E}\left[N_{t}^{\text {Pull }} \mid I_{t}\right]+\mathbf{E}\left[N_{t}^{\text {Push }} \mid I_{t}\right] \leqslant(1+4 \cdot \mathbf{E}[R]) \cdot I_{t} .
$$

By using the law of total expectation, we conclude that $\mathbf{E}\left[I_{t}\right]<(1+4 \cdot \mathbf{E}[R])^{t}$. If we set $T=c \cdot \log n$, where $c>0$ is a small constant, then

$$
\operatorname{Pr}\left[I_{T} \geqslant \sqrt{n}\right] \leqslant \frac{\mathbf{E}\left[I_{T}\right]}{\sqrt{n}} \leqslant \frac{(1+4 \cdot \mathbf{E}[R])^{T}}{\sqrt{n}}=o(1) .
$$

So with probability $1-o(1)$, we need at least $c \cdot \log n$ rounds to inform all nodes.

\section{References}

[1] P. Berenbrink, R. Elsässer, and T. Friedetzky. Efficient randomised broadcasting in random regular networks with applications in peer-to-peer systems. In Proc. 27th Symp. Principles of Distributed Computing (PODC), pages 155-164, 2008.

[2] N. Berger, C. Borgs, J. Chayes, and A. Saberi. On the Spread of Viruses on the Internet. In Proc. 16th Symp. Discrete Algorithms (SODA), pages 301-310, 2005.

[3] S. Boyd, A. Ghosh, B. Prabhakar, and D. Shah. Randomized gossip algorithms. IEEE Transactions on Information Theory, 52(6):2508-2530, 2006.

[4] K. Censor-Hillel and H. Shachnai. Partial information spreading with application to distributed maximum coverage. In Proc. 29th Symp. Principles of Distributed Computing (PODC), pages 161-170, 2010.

[5] F. Chierichetti, S. Lattanzi, and A. Panconesi. Rumor spreading in social networks. In Proc. 36th Intl. Coll. Automata, Languages and Programming (ICALP), pages 375-386, 2009.

[6] F. Chierichetti, S. Lattanzi, and A. Panconesi. Almost tight bounds for rumour spreading with conductance. In Proc. 42nd Symp. Theory of Computing (STOC), pages 399-408, 2010. 
[7] A. E. F. Clementi, F. Pasquale, and R. Silvestri. Opportunistic manets: Mobility can make up for low transmission power. IEEE/ACM Transaction on Networking, 21(2):610-620, 2013.

[8] A. Demers, D. Greene, C. Hauser, W. Irish, J. Larson, S. Shenker, H. Sturgis, D. Swinehart, and D. Terry. Epidemic algorithms for replicated database maintenance. In Proc. 6th Symp. Principles of Distributed Computing (PODC), pages $1-12,1987$.

[9] B. Doerr and M. Fouz. Asymptotically optimal randomized rumor spreading. In Proc. 38th Intl. Coll. Automata, Languages and Programming (ICALP), pages 502513, 2011.

[10] B. Doerr, M. Fouz, and T. Friedrich. Social networks spread rumors in sublogarithmic time. In Proc. 43th Symp. Theory of Computing (STOC), pages 21-30, 2011.

[11] B. Doerr, M. Fouz, and T. Friedrich. Asynchronous rumor spreading in preferential attachment graphs. In Proc. 13th Scandinavian Workshop Algorithm Theory (SWAT), pages 307-315, 2012.

[12] D. Dubhashi and A. Panconesi. Concentration of Measure for the Analysis of Randomized Algorithms. Cambridge University Press, 2009.

[13] R. Elsässer and T. Sauerwald. On the runtime and robustness of randomized broadcasting. In Proc. 17th Intl. Symp. Algorithms and Computation, (ISAAC), pages 349-358, 2006.

[14] R. Elsässer and T. Sauerwald. The power of memory in randomized broadcasting. In Proc. 19th Symp. Discrete Algorithms (SODA), pages 218-227, 2008.

[15] U. Feige, D. Peleg, P. Raghavan, and E. Upfal. Randomized broadcast in networks. Random Structures and Algorithms, 1(4):447-460, 1990.

[16] N. Fountoulakis, A. Huber, and K. Panagiotou. Reliable broadcasting in random networks and the effect of density. In Proc. 29th IEEE Conf. Computer Communications (INFOCOM), pages 2552-2560, 2010.

[17] N. Fountoulakis and K. Panagiotou. Rumor spreading on random regular graphs and expanders. In Proc. 14th Intl. Workshop on Randomization and Comput. (RANDOM), pages 560-573, 2010.

[18] N. Fountoulakis, K. Panagiotou, and T. Sauerwald. Ultra-fast rumor spreading in social networks. In Proc. 23th Symp. Discrete Algorithms (SODA), pages 1642-1660, 2012 .

[19] A. Frieze and G. Grimmett. The shortest-path problem for graphs with random-arclengths. Discrete Applied Mathematics, 10:57-77, 1985.

[20] G. Giakkoupis. Tight bounds for rumor spreading in graphs of a given conductance. In Proc. 28th Symp. Theoretical Aspects of Computer Science (STACS), pages 57-68, 2011.

[21] G. Giakkoupis. Tight bounds for rumor spreading with vertex expansion. CoRR, abs/1302.6243, 2013. 
[22] G. Giakkoupis and T. Sauerwald. Rumor spreading and vertex expansion. In Proc. 23th Symp. Discrete Algorithms (SODA), pages 1623-1641, 2012.

[23] M. Harchol-Balter, F. T. Leighton, and D. Lewin. Resource discovery in distributed networks. In Proc. 18th Symp. Principles of Distributed Computing (PODC), pages 229-237, 1999.

[24] R. Karp, C. Schindelhauer, S. Shenker, and B. Vöcking. Randomized Rumor Spreading. In Proc. 41st Symp. Foundations of Computer Science (FOCS), pages 565-574, 2000 .

[25] D. Kempe, A. Dobra, and J. Gehrke. Gossip-based computation of aggregate information. In Proc. 44th Symp. Foundations of Computer Science (FOCS), pages 482-491, 2003.

[26] C. McDiarmid. Concentration. In M. Habib, C. McDiarmid, J. Ramirez-Alfonsin, and B. Reed, editors, Probabilistic Methods for Algorithmic Discrete Mathematics, pages 195-243. Springer-Verlag, 1998.

[27] D. Mosk-Aoyama and D. Shah. Fast distributed algorithms for computing separable functions. IEEE Transactions on Information Theory, 54(7):2997-3007, 2008.

[28] Y. Peres, A. Sinclair, P. Sousi, and A. Stauffer. Mobile geometric graphs: Detection, coverage and percolation. In Proc. 22nd Symp. Discrete Algorithms (SODA), pages 412-428, 2011.

[29] B. Pittel. On spreading a rumor. SIAM Journal on Applied Mathematics, 47(1):213223, 1987.

[30] R. van Renesse, Y. Minsky, and M. Hayden. A gossip-style failure detection service. In Proc. 15th IFIP Intl. Conf. Distributed Systems Platforms (Middleware'98), pages 55-70, 1998. 